\title{
Genome-wide identification, phylogenetic and expression analysis of the heat shock transcription factor family in bread wheat (Triticum aestivum L.)
}

Min Zhou ${ }^{1,2,3}$, Shigang Zheng ${ }^{1}$, Rong Liu ${ }^{1,2}$, Jing Lu ${ }^{1,2}$, Lu Lu$^{1}$, Chihong Zhang ${ }^{1}$, Zehou Liu', Congpei Luo ${ }^{1,2}$, Lei Zhang ${ }^{1}$, Levi Yant ${ }^{3}$ and Yu Wu ${ }^{1 *}$

\begin{abstract}
Background: Environmental toxicity from non-essential heavy metals such as cadmium (Cd), which is released from human activities and other environmental causes, is rapidly increasing. Wheat can accumulate high levels of $\mathrm{Cd}$ in edible tissues, which poses a major hazard to human health. It has been reported that heat shock transcription factor A $4 a(H s f A 4 a)$ of wheat and rice conferred Cd tolerance by upregulating metallothionein gene expression. However, genome-wide identification, classification, and comparative analysis of the Hsf family in wheat is lacking. Further, because of the promising role of Hsf genes in Cd tolerance, there is need for an understanding of the expression of this family and their functions on wheat under $\mathrm{Cd}$ stress. Therefore, here we identify the wheat TaHsf family and to begin to understand the molecular mechanisms mediated by the Hsf family under Cd stress.

Results: We first identified 78 putative Hsf homologs using the latest available wheat genome information, of which 38 belonged to class A, 16 to class B and 24 to class C subfamily. Then, we determined chromosome localizations, gene structures, conserved protein motifs, and phylogenetic relationships of these TaHsfs. Using RNA sequencing data over the course of development, we surveyed expression profiles of these TaHsfs during development and under different abiotic stresses to characterise the regulatory network of this family. Finally, we selected 13 TaHsf genes for expression level verification under Cd stress using qRT-PCR.

Conclusions: To our knowledge, this is the first report of the genome organization, evolutionary features and expression profiles of the wheat Hsf gene family. This work therefore lays the foundation for targeted functional analysis of wheat Hsf genes, and contributes to a better understanding of the roles and regulatory mechanism of wheat Hsfs under $\mathrm{Cd}$ stress.
\end{abstract}

Keywords: Wheat, Hsf, Genome-wide analysis, Expression profiles

\section{Background}

Heat shock proteins (HSPs) perform important roles not only in cellular protection against stress-related damage, but also in the regular folding, intracellular distribution, and degradation of proteins. These functions facilitate organismal survival under stressful conditions $[1,2]$. Heat shock transcription factors ( $H s f s)$ modulate the

\footnotetext{
*Correspondence: wuyugood@126.com

${ }^{1}$ Chengdu Institute of Biology, Chinese Academy of Sciences, No.9, section 4 of South RenMin Road, Wuhou District, Chengdu 610041, Sichuan, China Full list of author information is available at the end of the article
}

expression of HSPs, and participate in various aspects of protein homeostasis, such as refolding, assembly and transporting damaged proteins, which sustain protein stability [3-7]. Hsfs share a core structure consisting of an N-terminal DNA binding domain (DBD) and an adjacent bipartite oligomerization domain (HR-A/B) $[6,8]$. Some $H s f s$ also share a leucine-rich nuclear export signal (NES) for nuclear export, a nuclear localization signal (NLS) essential for nuclear import,, and short peptide motifs (AHA motifs) for activator functions [9-12]. Based on the characteristics of their HR-A/B domain 
and phylogenetic comparisons, plant Hsf genes may be classified into three broad groups: A, B and C $[6,8]$. The HR-A/B regions of class B $H s f s$ are relatively compact, not including any insertions, while all class $\mathrm{A}$ and class $\mathrm{C}$ HSFs have an outspread HR-A/B region due to an insertion of 21 (class A) and seven (class C) amino acid residues [6]. This classification is also supported by differences in the flexible linkers between the DBD domain and HR-A/B domain, which consists of 9 to 39,50 to 78, and 14 to 49 amino acid residues in class $A, B$ and $C$ $H s f s$, respectively $[6,9]$. Recent studies indicate that $H s f s$ are engaged in plant development and growth, as well as in response to abiotic stresses such as salt, cold, drought and cadmium challenge [7, 9, 13-19]. For example, $H s f A 9$ is related to seed maturation and embryogenesis in sunflowers and Arabidopsis [14-16]. HsfA4a is involved in cadmium tolerance in wheat [19]. Due to the essential modulatory functions of $H s f$ genes in plants [16-18], the Hsf gene family has been studied in the models Arabidopsis thaliana and rice (Oryza sativa), and nonmodels such as poplar (Popupus trichocarpa), maize (Zea mays), and apple (Malus domestica) [5, 6, 9, 20-22]. However, the Hsf gene family in the bread wheat (Triticum aestivum) has not been systematically examined.

Bread wheat is one of the most widely grown and consumed crops worldwide [23]. Bread wheat is hexaploid ( $2 \mathrm{n}=6 \mathrm{x}=42$; AABBDD genome), originating from two amphiploidization events: the first hybridization producing the tetraploid wheat species $(2 \mathrm{n}=4 \mathrm{x}=28$, genome AABB) was between the Triticum urartu $(2 \mathrm{n}=2 \mathrm{x}=14$, genome AA) and presumably Aegilops speltoides, belonging to the section Sitopsis $(2 \mathrm{n}=2 \mathrm{x}=14$, genome SS); the second hybridization was between the tetraploid wheat and Aegilops tauschii $(2 \mathrm{n}=2 \mathrm{x}=14$, genome DD) $[24,25]$. Therefore, bread wheat has a huge and highly complex genome with three subgenomes (A, B and D) and $\sim 17 \mathrm{~Gb}$ total size [26], leading to great challenges for genomic studies. Recently, however, a quality draft genome of hexaploid 'Chinese Spring' wheat has provided the foundation upon which we can investigate wheat gene families and to clearly recognize homologous gene copies in these three sub-genomes [27]. Further, it has allowed the study of interactions of loci during polyploidization and the retention and dispersion of homologous gene $[28,29]$.

Here we first perform an in silico genome-wide study to comprehensively identify members of the wheat $H s f$ gene family. Next, to characterize evolutionary and functional features, we determine chromosome locations, gene structures, conserved protein domains, phylogenetic relationships and expression profiles for this family. Our study provides a foundation for downstream targeted functional investigation of wheat $H s f$ genes, and will be allow for better understanding of the molecular mechanisms by which $H s f s$ regulate in growth, development and stress resilience in wheat.

\section{Results \\ Genome-wide identification and classification of $\mathrm{Hsf}$ family in wheat}

Through the availability of the genome sequence, it is possible for the first time to identify all the $H s f$ family members in wheat. In this study, we identified a total of 78 genes as $H s f$ members in the wheat genome, designating the predicted wheat $H s f$ genes TaHsf1 to TaHsf78. Members of the Hsf gene family have been broadly subdivided into Classes $\mathrm{A}, \mathrm{B}$, and $\mathrm{C}$ according to differences in the length of the flexible linkers between the A and $\mathrm{B}$ parts of the HR-A/B regions. In the TaHsf gene family, 38, 16 and 24 genes were accordingly assigned to Classes $\mathrm{A}, \mathrm{B}$ and $\mathrm{C}$, respectively. Within the A clade, 8 distinct subclades (A1, to A8) were resolved. The B-type $H s f$ genes were grouped into a separate clade subdivided into three groups (B1, B2 and B4). And the C-type genes were subdivided into two groups $(\mathrm{C} 1$ and $\mathrm{C} 2)$. We further performed a BLASTN search against the wheat expressed sequence tag (EST) using the 78 identified $H s f s$ as queries to verify the existence and completeness of this set of wheat Hsfs. Results showed that most of the TaHsfs were supported by EST hits except $2 H s f s$ (TaHsf57 and TaHsf75). We speculated these 2 unsupportted TaHsfs might not be expressed under any the assayed conditions or may be expressed at very low level that cannot be easily detected. Among the supported TaHsf genes, TaHsf8 has the largest number of EST hits, with 49, followed by TaHsf 21 and TaHsf27 with 48 and 30 hists, respectively. Chromosome localization analysis found that 4 TaHsfs did not have corresponding chromosomal locations, and that the remaining 74 TaHsf genes were distributed on all of the 21 wheat chromosomes. Chromosome 3B contained the most $H s f$ genes with 8 , followed by $4 \mathrm{~B}, 5 \mathrm{~A}$ and $5 \mathrm{D}$, with each harboring 6 , then $3 \mathrm{~A}$ with 5 , and finally $6 \mathrm{~A}, 6 \mathrm{~B}$ and $6 \mathrm{D}$ with one each. The predicted lengths of the putative TaHsf proteins ranged from 209 to 701 amino acids, with the molecular weights $(\mathrm{Mw})$ ranging from 22.72 to 73.92 $\mathrm{kDa}$ and theoretical isoelectric points (PI) ranging from 4.67 to 9.50 (Table 1).

\section{Conserved domains analysis of TaHsf}

We identified five conserved domains by sequence alignment approaches (Table 2). All the TaHsf predicted proteins contained a highly conserved DBD domain, forming with a three helical bundles ( $\mathrm{H} 1, \mathrm{H} 2$ and $\mathrm{H} 3)$ and four-stranded antiparallel $\beta$-sheet in their $\mathrm{N}$ terminal regions. However, within the Hsf family, the length of the DBD domain was quite different. We then used the MARCOIL tool to detect the presence of a 
Table 1 The list of the putative wheat Hsf genes

\begin{tabular}{|c|c|c|c|c|c|c|c|c|c|}
\hline Names & Ensemble Gene ID & Chromosome location & $\begin{array}{l}\text { EST } \\
\text { count }\end{array}$ & $\begin{array}{l}\text { length } \\
\text { (bp) }\end{array}$ & Exons & Introns & $\begin{array}{l}\text { Amino acid length } \\
\text { (aa) }\end{array}$ & $\mathrm{Pl}$ & $\begin{array}{l}\mathrm{MW} \\
(\mathrm{kDa})\end{array}$ \\
\hline TaHsfl & Trae_4AL_8577C148B & scaffold_288809_4AL: 49,335-56,655 & 26 & 7321 & 3 & 2 & 521 & 4.94 & 57.34 \\
\hline TaHsf2 & Trae_5BL_E15759DAD & scaffold_404129_5BL: 211,116-217,536 & 26 & 6421 & 2 & 1 & 471 & 5.18 & 52.89 \\
\hline TaHsf3 & Trae_5DL_B1D24781B1 & scaffold_433347_5DL: 108,916-114,305 & 28 & 5390 & 2 & 1 & 487 & 4.95 & 54.60 \\
\hline TaHsf4 & Trae_5AL_16AD8DEEC & scaffold_375092_5AL: 45,746-49,544 & 4 & 3799 & 2 & 1 & 346 & 5.45 & 38.98 \\
\hline TaHsf5 & Trae_5DL_6EB179C88 & scaffold_434875_5DL: 17,703-21,445 & 8 & 3743 & 2 & 1 & 348 & 5.39 & 38.90 \\
\hline TaHsf6 & nd & scaffold_640974_U: 63,006-67,190 & 6 & 4185 & 2 & 1 & 353 & 5.59 & 39.72 \\
\hline TaHsf7 & Trae_2AS_CF07F4EC2 & scaffold_113503_2AS: 55,860-61,955 & 26 & 6096 & 2 & 1 & 413 & 4.99 & 45.60 \\
\hline TaHsf8 & Trae_2BS_ECF9B4EB4 & scaffold_148328_2BS: 27,356-32,557 & 49 & 5202 & 2 & 1 & 405 & 5.06 & 44.92 \\
\hline TaHsfg & Trae_2DS_B6872CB84 & scaffold_177319_2DS: 131,495-137,254 & 27 & 5760 & 2 & 1 & 412 & 4.85 & 45.43 \\
\hline TaHsfio & Trae_3AL_E15419B88 & scaffold_194616_3AL: 22,466-26,620 & 2 & 4155 & 2 & 1 & 314 & 6.14 & 35.42 \\
\hline TaHsfil & TRAES3BF002300100CFD & scaffold_221589_3B: 97,736-102,700 & 4 & 4965 & 3 & 2 & 396 & 5.09 & 43.99 \\
\hline TaHsfi2 & nd & scaffold_379543_5AL: 6399-8897 & 10 & 2499 & 3 & 2 & 372 & 5.37 & 41.13 \\
\hline TaHsfl3 & nd & scaffold_433195_5DL: 110,106-113,560 & 9 & 3455 & 2 & 1 & 377 & 5.42 & 41.59 \\
\hline TaHsfi4 & nd & scaffold_116363_2AS: 3667-6615 & 7 & 2949 & 2 & 1 & 467 & 6.06 & 51.62 \\
\hline TaHsf15 & Trae_2AS_53BFA14C7 & scaffold_114504_2AS: 30,086-34,710 & 7 & 4625 & 4 & 3 & 502 & 5.95 & 55.44 \\
\hline TaHsfi6 & Trae_2BS_1484A7516 & scaffold_146118_2BS: 176,336-179,440 & 7 & 3105 & 2 & 1 & 475 & 5.94 & 52.08 \\
\hline TaHsfl7 & Trae_2DS_070CE3D50 & scaffold_177422_2DS: 92,871-96,205 & 8 & 3335 & 2 & 1 & 499 & 5.7 & 54.78 \\
\hline TaHsfli & Trae_3AL_463ABD4BF & scaffold_196554_3AL: 30,462-32,936 & 15 & 2475 & 2 & 1 & 432 & 5.36 & 48.37 \\
\hline TaHsf19 & TRAES3BF029100010CFD & scaffold_223991_3B: 37,660-40,120 & 15 & 2461 & 2 & 1 & 441 & 5.18 & 49.46 \\
\hline TaHsf20 & Trae_3DL_8FD0F859B & scaffold_249383_3DL: 45,727-48,075 & 16 & 2349 & 2 & 1 & 433 & 5.35 & 48.45 \\
\hline TaHsf21 & Trae_1AL_7D6DC73FC & scaffold_001183_1AL: 39,381-43,295 & 48 & 3915 & 3 & 2 & 448 & 4.91 & 50.25 \\
\hline TaHsf22 & nd & scaffold_031159_1BL: 70,097-72,255 & 9 & 2159 & 3 & 2 & 445 & 4.94 & 49.92 \\
\hline TaHsf23 & nd & scaffold_061383_1DL: 26,931-29,065 & 8 & 2135 & 3 & 2 & 442 & 5.11 & 49.70 \\
\hline TaHsf24 & Trae_6AS_1537629B3 & scaffold_487059_6AS: 7273-11,032 & 7 & 3760 & 2 & 1 & 458 & 5.21 & 49.87 \\
\hline TaHsf25 & Trae_6BS_25E162197 & scaffold_513816_6BS: 34,066-37,539 & 8 & 3474 & 2 & 1 & 455 & 5.33 & 49.92 \\
\hline TaHsf26 & Trae_6DS_C59B6322F & scaffold_543918_6DS: 1556-5556 & 7 & 4001 & 2 & 1 & 458 & 5.16 & 49.86 \\
\hline TaHsf27 & Trae_1AL_A4B5C1474 & scaffold_003124_1AL: 28,946-32,101 & 30 & 3156 & 5 & 4 & 368 & 5 & 41.70 \\
\hline TaHsf28 & Trae_1BL_5D8D6B865 & scaffold_031443_1BL: 79,599-83,003 & 27 & 3405 & 4 & 3 & 364 & 4.89 & 41.02 \\
\hline TaHsf29 & Trae_1DL_B5A84E4C8 & scaffold_061579_1DL: 62,790-66,102 & 29 & 3313 & 4 & 3 & 370 & 5.03 & 42.03 \\
\hline TaHsf30 & Trae_4AS_52EB860E7 & scaffold_307193_4AS: 64,786-67,745 & 13 & 2960 & 2 & 1 & 341 & 5.07 & 39.63 \\
\hline TaHsf31 & Trae_4BL_2E125A702 & scaffold_321575_4BL: 50,126-53,221 & 12 & 3096 & 2 & 1 & 341 & 5.07 & 39.59 \\
\hline TaHsf32 & Trae_4DL_AF19ABC7D & scaffold_342984_4DL: 44,562-50,805 & 13 & 6244 & 4 & 3 & 341 & 5.02 & 39.49 \\
\hline TaHsf33 & nd & scaffold_559301_7AL: 9972-11,835 & 4 & 1864 & 5 & 4 & 310 & 4.67 & 33.78 \\
\hline TaHsf34 & nd & scaffold_579527_7BL: 16,166-18,111 & 5 & 1946 & 4 & 3 & 351 & 4.94 & 37.90 \\
\hline TaHsf35 & nd & scaffold_605087_7DL: 18,736-21,007 & 5 & 2272 & 4 & 3 & 351 & 4.82 & 37.98 \\
\hline TaHsf36 & Trae_4AS_02B607421 & scaffold_306492_4AS: 132,616-136,430 & 10 & 3815 & 4 & 3 & 383 & 5.22 & 42.84 \\
\hline TaHsf37 & Trae_4BL_542B1DA85 & scaffold_322416_4BL: 4327-8215 & 9 & 3889 & 4 & 3 & 384 & 5.3 & 42.87 \\
\hline TaHsf38 & Trae_4DL_EE941086E & scaffold_344014_4DL: 13,486-17,310 & 9 & 3825 & 4 & 3 & 384 & 5.3 & 42.92 \\
\hline TaHsf39 & Trae_5AL_D369204D3 & scaffold_374310_5AL: 146,720-151,848 & 28 & 5129 & 2 & 1 & 298 & 9.5 & 32.14 \\
\hline TaHsf40 & Trae_5BL_F80E01D65 & scaffold_404669_5BL: 141,516-147,139 & 27 & 5624 & 2 & 1 & 298 & 9.31 & 32.28 \\
\hline TaHsf41 & Trae_5DL_431CCA490 & scaffold_433651_5DL: 31,056-36,542 & 28 & 5487 & 2 & 1 & 298 & 9.2 & 32.06 \\
\hline TaHsf42 & Trae_2AL_D3B2C21A7 & scaffold_094650_2AL: 33,644-35,170 & 2 & 1527 & 2 & 1 & 295 & 6.12 & 31.99 \\
\hline TaHsf43 & nd & scaffold_712376_U: 1-715 & 1 & 715 & 2 & 1 & 209 & 9.5 & 22.72 \\
\hline
\end{tabular}


Table 1 The list of the putative wheat Hsf genes (Continued)

\begin{tabular}{|c|c|c|c|c|c|c|c|c|c|}
\hline Names & Ensemble Gene ID & Chromosome location & $\begin{array}{l}\text { EST } \\
\text { count }\end{array}$ & $\begin{array}{l}\text { length } \\
\text { (bp) }\end{array}$ & Exons & Introns & $\begin{array}{l}\text { Amino acid length } \\
\text { (aa) }\end{array}$ & $\mathrm{Pl}$ & $\begin{array}{l}\text { MW } \\
(\mathrm{kDa})\end{array}$ \\
\hline TaHsf44 & nd & scaffold_019033_1AS: 12,760-16,705 & 25 & 3946 & 3 & 2 & 404 & 4.9 & 42.04 \\
\hline TaHsf45 & Trae_5BL_FCB1625F3 & scaffold_404935_5BL: 109,416-113,225 & 27 & 3810 & 3 & 2 & 701 & 9.22 & 73.92 \\
\hline TaHsf46 & nd & scaffold_433530_5DL: 41,946-43,807 & 26 & 1862 & 2 & 1 & 397 & 4.89 & 41.11 \\
\hline TaHsf47 & Trae_7AS_937121AF8 & scaffold_570040_7AS: 14,527-16,335 & 6 & 1809 & 2 & 1 & 374 & 5.44 & 40.45 \\
\hline TaHsf48 & Trae_7BS_03F39ED94 & scaffold_592325_7BS: 110,144-112,895 & 6 & 2752 & 3 & 2 & 374 & 5.33 & 40.33 \\
\hline TaHsf49 & Trae_7DS_10A9C68FA & scaffold_621446_7DS: 14,666-16,580 & 6 & 1915 & 2 & 1 & 367 & 5.5 & 39.79 \\
\hline TaHsf50 & Trae_2DS_01A0E5F7A & scaffold_178567_2DS: 15,585-18,518 & 4 & 2934 & 2 & 1 & 320 & 6.55 & 35.31 \\
\hline TaHsf51 & nd & scaffold_642758_U: 53,288-55,875 & 5 & 2587 & 2 & 1 & 320 & 6.66 & 35.26 \\
\hline TaHsf52 & nd & scaffold_374067_5AL: 30,626-32,510 & 7 & 1885 & 2 & 1 & 388 & 7.85 & 41.35 \\
\hline TaHsf53 & nd & scaffold_404268_5BL: 201,437-203,325 & 8 & 1889 & 2 & 1 & 388 & 7.89 & 41.46 \\
\hline TaHsf54 & nd & scaffold_433663_5DL: 11,036-12,820 & 8 & 1785 & 2 & 1 & 388 & 8.42 & 41.39 \\
\hline TaHsf55 & nd & scaffold_201352_3AL: 447-1655 & 0 & 1209 & 3 & 2 & 277 & 5.54 & 31.15 \\
\hline TaHsf56 & nd & scaffold_194514_3AL: 70,656-72,591 & 2 & 1936 & 2 & 1 & 294 & 6.26 & 32.57 \\
\hline TaHsf57 & nd & scaffold_220888_3B: 91,006-92,356 & 0 & 1351 & 2 & 1 & 322 & 5.46 & 35.55 \\
\hline TaHsf58 & TRAES3BF021000010CFD & scaffold_220882_3B: 116,126-117,710 & 2 & 1585 & 2 & 1 & 325 & 5.94 & 35.72 \\
\hline TaHsf59 & nd & scaffold_249994_3DL: 60,736-62,250 & 2 & 1515 & 2 & 1 & 321 & 6.16 & 35.38 \\
\hline TaHsf60 & nd & scaffold_249450_3DL: 110,687-117,555 & 16 & 6869 & 3 & 2 & 225 & 7.11 & 25.63 \\
\hline TaHsf61 & nd & scaffold_193607_3AL: 163,384-164,530 & 13 & 1147 & 2 & 1 & 236 & 6.91 & 26.05 \\
\hline TaHsf62 & TRAES3BF005500020CFD & scaffold_223354_3B: 26,214-27,330 & 14 & 1117 & 2 & 1 & 227 & 8.35 & 24.69 \\
\hline TaHsf63 & nd & scaffold_250779_3DL: 25,456-26,755 & 11 & 1300 & 2 & 1 & 241 & 8.76 & 26.40 \\
\hline TaHsf64 & TRAES_3BF025700020CFD_c1 & scaffold_231430_3B: 2326-3400 & 4 & 1075 & 1 & 0 & 237 & 5.11 & 26.12 \\
\hline TaHsf65 & nd & scaffold_223198_3B: 70,994-72,095 & 2 & 1102 & 2 & 1 & 237 & 5.99 & 26.61 \\
\hline TaHsf66 & TRAES3BF025700030CFD & scaffold_224063_3B: 9915-11,221 & 27 & 1307 & 1 & 0 & 274 & 6.98 & 29.98 \\
\hline TaHsf67 & Trae_4BL_86572BB6D & scaffold_321958_4BL: 10,751-11,965 & 9 & 1215 & 1 & 0 & 264 & 5.43 & 29.06 \\
\hline TaHsf68 & Trae_4BL_F6C3B5069 & scaffold_320289_4BL: 21,946-23,120 & 25 & 1175 & 1 & 0 & 275 & 8.4 & 30.21 \\
\hline TaHsf69 & Trae_4BL_5091DE58E & scaffold_320289_4BL: 33,386-34,490 & 2 & 1105 & 1 & 0 & 257 & 4.88 & 28.50 \\
\hline TaHsf70 & nd & scaffold_320675_4BL: 112,161-113,540 & 5 & 1380 & 2 & 1 & 273 & 5.7 & 29.45 \\
\hline TaHsf71 & nd & scaffold_344468_4DL: 19,506-20,710 & 14 & 1205 & 1 & 0 & 276 & 6.46 & 30.27 \\
\hline TaHsf72 & Trae_4DL_FA07D8414 & scaffold_343739_4DL: 22,666-23,885 & 4 & 1220 & 1 & 0 & 276 & 5.32 & 29.85 \\
\hline TaHsf73 & nd & scaffold_376864_5AL: 4896-6010 & 4 & 1115 & 1 & 0 & 273 & 6.15 & 30.17 \\
\hline TaHsf74 & nd & scaffold_375679_5AL: 69,576-70,900 & 15 & 1325 & 1 & 0 & 229 & 5.08 & 25.56 \\
\hline TaHsf75 & nd & scaffold_641118_U: 187,271-188,375 & 0 & 1105 & 2 & 1 & 268 & 5.69 & 29.99 \\
\hline TaHsf76 & Trae_7AL_6931AA68B & scaffold_558532_7AL: 22,876-24,495 & 13 & 1620 & 2 & 1 & 266 & 6.44 & 28.23 \\
\hline TaHsf77 & nd & scaffold_577398_7BL: 12,506-14,356 & 14 & 1851 & 2 & 1 & 244 & 5.61 & 26.12 \\
\hline TaHsf78 & nd & scaffold_609477_7DL: 1-1636 & 16 & 1636 & 2 & 1 & 263 & 6.11 & 28.04 \\
\hline
\end{tabular}

property of the HR-A/B, the coiled-coil structure characteristic of leucine zipper-type protein interaction domains. We found that most of the TaHsfs proteins consisted of NES and NLS domains, which are vital for shuttling $H s f s$ between the nucleus and cytoplasm. As was expected in the A-type TaHsfs, additional sequence comparisons identified AHA domain in the middle of the C-terminal activation domains. By contrast, these domains were not detected in the B- and C-type TaHsfs.
To further predict and verify domains in the TaHsfs proteins, we used the Multiple EM for Motif Elicitation (MEME) motif search tool. Using this, we found thirty corresponding consensus motifs (Additional file 1: Figure S1, Additional file 2). Compared with class B and $\mathrm{C}$, the members of class A contained the greatest number of conserved motifs (22), with the majority (12) detected in TaHsf1 and TaHsf3. The conserved motifs 1, 2, 4, 5, 816 represented the DBD domain. Motif 1 was 
Table 2 Functional domains of TaHsfs

\begin{tabular}{|c|c|c|c|c|c|c|}
\hline Names & Protein type (A-B-C) & DBD & $H R-A / B$ & NLS & NES & $\mathrm{AHA}$ \\
\hline TaHsfl & A1a & $38-128$ & $163-227$ & (245)RRIVAANKKRR & (508)LTEQMGLL & AHA2(464)DSFWEQFLCA \\
\hline TaHsf2 & A1a & $1-73$ & $109-173$ & (191)RRIVAANKKRR & (458)LTEQMGLL & AHA2(414)DSFWEQFLCA \\
\hline TaHsf3 & Ala & $1-91$ & $125-189$ & (207)RRIVAANKKRR & (474)LTEQMGLL & AHA2(430)DSFWEQFLCA \\
\hline TaHsf4 & $\mathrm{A} 2 \mathrm{a}$ & $38-128$ & $143-207$ & (223)RKELEDAISNKRRRR & nd & AHA1(313)DDFWEDLL \\
\hline TaHsf5 & $\mathrm{A} 2 \mathrm{a}$ & $40-130$ & $145-209$ & (225)RKELEDAISNKRRRR & nd & AHA1(315)DDFWEDLL \\
\hline TaHsf6 & $\mathrm{A} 2 \mathrm{a}$ & $45-135$ & $150-214$ & (230)RKELEDAISNKRRRR & nd & AHA(320)DDFWEDLL \\
\hline TaHsf7 & $\mathrm{A} 2 \mathrm{~b}$ & $43-133$ & $149-213$ & (229)RKELHDAISKKRRRR & (400)KMGYL & AHA1(370)DNFWEELL \\
\hline TaHsf8 & $A 2 b$ & $44-134$ & $150-214$ & (230)SKELHDAISKKRRRR & (392)KMGYF & AHA1(362)DNFWEGLL \\
\hline TaHsf9 & $\mathrm{A} 2 \mathrm{~b}$ & $43-133$ & $149-213$ & (229)RKELHDAISKKRRRR & (399)KMGYL & AHA1(369)DNFWEELL \\
\hline TaHsfio & $A 2 b$ & $42-132$ & $148-212$ & (228)RKELHDAMSKKRRRS & nd & nd \\
\hline TaHsfil & $A 2 b$ & $41-131$ & $147-211$ & (227)RKELHDAMSKKRRRS & nd & AHA1(353)DDFWEELM \\
\hline TaHsfl2 & A2e & $66-156$ & $178-242$ & (260)RKELAEALLSKKRGR & nd & AHA1(314)ESFWKELL \\
\hline TaHsfl3 & A2e & $66-156$ & $180-244$ & (262)RKELAEALLSKKRGR & nd & AHA1(320)ESFWKELL \\
\hline TaHsfi4 & $\mathrm{A} 3$ & 49-139 & $175-221$ & (248)RVKRKFLKHV & nd & nd \\
\hline TaHsf15 & $\mathrm{A} 3$ & $84-174$ & $210-256$ & (283)RVKRKFLKHV & nd & nd \\
\hline TaHsfl6 & $\mathrm{A} 3$ & $80-170$ & $206-252$ & (279)RVKRKFLKHV & nd & nd \\
\hline TaHsfi7 & A3 & $81-171$ & $207-253$ & (280)RVKRKFLKHV & nd & nd \\
\hline TaHsflis & A4a & $13-103$ & $126-183$ & (198)KKRR & (419)MTEKLGHL & AHA1(244)LNSLENFFKE AHA2(370)DGFWQQFLTE \\
\hline TaHsfl9 & A4a & $13-103$ & $126-183$ & (198)KKRR & (428)MTEKLGHL & AHA1(244)LNSLENFFKE AHA2(379)DGFWQQFLTE \\
\hline TaHsf2O & A4a & $13-103$ & $126-183$ & (198)KKRR & (420)MTKKLGHL & AHA1(244)LNSLENFFKE AHA2(370)DGFWQQFLTE \\
\hline TaHsf 21 & A4d & $25-115$ & 138-195 & (220)KKRR & (430)ITQQMGHL & AHA1(267)LVSMEKLVQR AHA2(386)DLFWERFLTD \\
\hline TaHsf22 & A4d & $23-113$ & $136-193$ & (219)KKRR & (432)ITEQMGHL & AHA1(267)LVSMEKLVRR AHA2(388)DLFWERFLTD \\
\hline TaHsf23 & A4d & $23-113$ & $136-193$ & (218)KKRR & (429)ITEQMGHL & AHA1(270)LVSMEKLVQR AHA2(385)DLFWERFLTD \\
\hline TaHsf24 & A5 & $20-111$ & $131-188$ & (199)KMAEASSMFADALHKK & nd & (414)DNFWEQFLTE \\
\hline TaHsf25 & A5 & $20-111$ & $131-188$ & (199)KMAEASSMFADALHKK & nd & (414)DNFWEQFLTE \\
\hline TaHsf26 & A5 & $20-111$ & $131-188$ & (199)KMAEASSMFADALHKK & nd & (414)DNFWEQFLTE \\
\hline TaHsf27 & A6a & $52-142$ & $159-223$ & (238)KRKELEDAISKKRRR & (352)IDELGQQLGYL & (322)SDFWAELFSD \\
\hline TaHsf28 & A6a & $48-138$ & $155-219$ & (234)KRKELEDAISKKRRR & (348)IDELAQQLGYL & (318)NDFWAELFSD \\
\hline TaHsf29 & A6a & $54-144$ & $161-225$ & (240)KRKELEDAISKKRRR & (354)IDELAQQLGYL & (324)NDFWAELFSD \\
\hline TaHsf30 & A6b & $46-136$ & $153-217$ & (232)KLKDLEDGYPTKRRR & nd & (311)DDFWEELLSE \\
\hline TaHsf31 & A6b & $46-136$ & $153-217$ & (232)KLKDLEDGYPTKRRR & nd & (311)DDFWEELLSE \\
\hline TaHsf32 & A6b & $46-136$ & $153-217$ & (232)KLKDLEDAYSNKRRR & nd & (311)DDFWEELLSE \\
\hline TaHsf33 & $A 7 b$ & $47-138$ & $150-175$ & nd & nd & (246)TDMIWYELL \\
\hline TaHsf34 & $A 7 b$ & 49-139 & $173-223$ & nd & nd & (298)TDMIWYELL \\
\hline TaHsf35 & $A 7 b$ & $49-139$ & $173-223$ & nd & nd & (298)TDMIWYELL \\
\hline TaHsf36 & A8 & $37-127$ & $173-230$ & nd & nd & nd \\
\hline TaHsf37 & A8 & $37-127$ & $173-230$ & nd & nd & nd \\
\hline TaHsf38 & A8 & $37-127$ & $173-230$ & nd & nd & nd \\
\hline TaHsf39 & B1 & $27-117$ & $172-209$ & nd & nd & nd \\
\hline TaHsf4O & B1 & $30-120$ & $174-211$ & nd & nd & nd \\
\hline TaHsf41 & B1 & $30-120$ & $174-211$ & nd & nd & nd \\
\hline TaHsf42 & B2a & 13-103 & 157-193 & (223)KRSRE & nd & nd \\
\hline TaHsf43 & B2a & $26-116$ & 170-206 & nd & nd & nd \\
\hline TaHsf44 & $B 2 C$ & $42-132$ & $215-251$ & (321)KRARD & nd & nd \\
\hline
\end{tabular}


Table 2 Functional domains of TaHsfs (Continued)

\begin{tabular}{|c|c|c|c|c|c|c|}
\hline Names & Protein type $(A-B-C)$ & DBD & $\mathrm{HR}-\mathrm{A} / \mathrm{B}$ & NLS & NES & $\mathrm{AHA}$ \\
\hline TaHsf45 & $\mathrm{B} 2 \mathrm{C}$ & $176-266$ & $349-385$ & (455)KRARD & nd & nd \\
\hline TaHsf46 & $\mathrm{B} 2 \mathrm{C}$ & $42-132$ & $215-251$ & (321)KRARD & nd & nd \\
\hline TaHsf47 & $B 2 d$ & $32-122$ & $192-228$ & (300)KRMRH & nd & nd \\
\hline TaHsf48 & B2d & $32-122$ & $192-228$ & (300)KRMRH & nd & nd \\
\hline TaHsf49 & $B 2 d$ & $32-122$ & $192-228$ & (293)KRMRH & nd & nd \\
\hline TaHsf50 & $B 4 b$ & $40-130$ & $201-237$ & (299)KKKR & nd & nd \\
\hline TaHsf51 & B4b & 39-129 & $200-236$ & (299)KKKR & nd & nd \\
\hline TaHsf52 & $B 4 C$ & $26-117$ & $207-243$ & (336)PVGA & (362)LALENDDL & nd \\
\hline TaHsf53 & B4C & $26-117$ & $207-243$ & (336)PVGA & (362)LALESDDL & nd \\
\hline TaHsf54 & $B 4 C$ & $26-117$ & $207-243$ & (336)PVGA & (362)LALESDDL & nd \\
\hline TaHsf55 & C1a & $21-111$ & $121-164$ & nd & nd & nd \\
\hline TaHsf56 & C1a & $1-84$ & $121-171$ & nd & nd & nd \\
\hline TaHsf57 & C1a & $21-111$ & 154-197 & nd & nd & nd \\
\hline TaHsf58 & Cla & $25-115$ & 159-202 & nd & nd & nd \\
\hline TaHsf59 & $\mathrm{C} 1 \mathrm{a}$ & 25-115 & 159-202 & nd & nd & nd \\
\hline TaHsf60 & $\mathrm{Cla}$ & $21-111$ & 149-192 & nd & nd & nd \\
\hline TaHsf61 & $\mathrm{c} 1 \mathrm{~b}$ & 19-109 & $126-169$ & nd & nd & nd \\
\hline TaHsf62 & $\mathrm{C} 1 \mathrm{~b}$ & 19-109 & $131-174$ & nd & nd & nd \\
\hline TaHsf63 & $c 1 b$ & 19-109 & $131-174$ & nd & nd & nd \\
\hline TaHsf64 & $\mathrm{C} 2 \mathrm{a}$ & $1-75$ & $97-140$ & (168)KRPR & & \\
\hline TaHsf65 & $\mathrm{C} 2 \mathrm{a}$ & 24-114 & 134-177 & (202)KRPR & nd & nd \\
\hline TaHsf66 & $\mathrm{C} 2 \mathrm{a}$ & 19-109 & $132-175$ & (203)KRPR & nd & nd \\
\hline TaHsf67 & $\mathrm{C} 2 \mathrm{a}$ & $12-102$ & $124-167$ & (195)QRPR & nd & nd \\
\hline TaHsf68 & $\mathrm{C} 2 \mathrm{a}$ & $21-111$ & $135-178$ & (206)KRPR & nd & nd \\
\hline TaHsf69 & $\mathrm{C} 2 \mathrm{a}$ & 20-110 & $132-175$ & (203)KKPR & nd & nd \\
\hline TaHsf70 & C2a & 24-114 & 135-178 & (205)KRPR & nd & nd \\
\hline TaHsf71 & $\mathrm{C} 2 \mathrm{a}$ & $23-113$ & $135-178$ & (206)KRRR & nd & nd \\
\hline TaHsf72 & C2a & 24-114 & $141-184$ & (211)KRPR & nd & nd \\
\hline TaHsf73 & $\mathrm{C} 2 \mathrm{a}$ & $30-120$ & 143-186 & (207)NRPR & nd & nd \\
\hline TaHsf74 & $\mathrm{C} 2 \mathrm{a}$ & $1-84$ & $106-149$ & (177)KRPR & nd & nd \\
\hline TaHsf75 & $\mathrm{C} 2 \mathrm{a}$ & 23-113 & $132-175$ & (198)KRLR & nd & nd \\
\hline TaHsf76 & $\mathrm{c} 2 \mathrm{~b}$ & 15-105 & $132-175$ & (204)KRAR & nd & nd \\
\hline TaHsf77 & $c 2 b$ & $1-84$ & $97-153$ & nd & nd & nd \\
\hline TaHsf78 & $c 2 b$ & 13-103 & $129-172$ & (201)KRAR & nd & nd \\
\hline
\end{tabular}

DBD DND-binding domain, HR-A/B OD (oligomerisation domain), heptad pattern of hydrophobic amino acid residues; NLS: Nuclear localization signal, NES Nuclear export signal. AHA Activator motifs, aromatic (W, F, Y), larger hydrophobic $(\mathrm{L}, \mathrm{I}, \mathrm{V})$ and acidic $(\mathrm{E}, \mathrm{D})$ amino acid residues; Numbers in brackets reveals the position of the first amino acid present in the putative NLS, NES, and $\bar{A} H A$ in the C-terminal; nd: no domains detectable by sequence similarity

found in 77 members of TaHsf family (except for TaHsf33). Regarding coiled-coiled structures, motif 3 was detected in class A and class C TaHsfs family, while motif 7 was detected in class B. The conserved motifs $10,20,22,23,25,28,30$ were identified as NLS domains. Motifs 10 and 25 represented NLS domains in class A, whereas NLS domains were represented by motifs 20 , 23, 28 and 30 in class B, motifs 22 and 23 in class C. Motif 15 represented NES domains, and motif11 was identified as characteristic AHA domains. Thus through the combination of the two methods, predicted DBD domains and HR-A/B domains were observed in each TaHsfs and varied greatly in size and sequence.

\section{Phylogenetic analysis in wheat Hsf proteins}

To further evaluate the phylogenetic relationships amidst $H s f$ families, the $H s f$ conserved amino acid sequences (from the beginning of the DNA-binding domain to the 
end of the HR-A/B region) of 39 proteins from wheat (Triticum aestivum L.), 21 proteins from Arabidopsis (A. thaliana), 25 from rice (O. sativa), 24 from brachypodium (B. distachyon) and 30 from maize (Z. mays) were used to construct a phylogenetic tree (Fig. 1). According to this tree, class $H s f A$ showed the maximum number of subclasses among the three major groups, and contained eight smaller clusters of which five (A6, A2, A8, A1 and A7) were closer to class $H s f C$ than class HsfA3, A4 and A5. Two HsfA6 members from Arabidopsis (At5g43840 and At3g22830) were not clustered with the HsfA6 subclass from other plant species, but were closer to the HsfA7 subclass. Brachypodium Hsfs were closer to wheat Hsf proteins compared with Arabidopsis, maize and rice, which was in line with the botanical classification.

\section{Genome distribution and gene duplication of TaHsf gene family}

We next determined chromosomal locations of TaHsf genes by leveraging the available wheat genome annotation information (Fig. 2). A total of 25, 26 and 23 TaHsf genes are found in the $\mathrm{A}, \mathrm{B}$ and $\mathrm{D}$ sub-genomes, respectively $(\mathrm{B}>\mathrm{A}>\mathrm{D})$. The distribution of Hsf genes was not even across the chromosomes. There were 7, 9, 17, 13, 16, 3 and 9 genes in the group 1 to group 7 chromosomes, which reveal obvious differences between group 3, 4, 5 and other four groups. Chromosome 3B had the highest number of $H s f$ genes with 8, while chromosome 6A, 6B and 6D all had only one $H s f$ gene eachs. These results suggest that $H s f$ gene duplication events may have happened in wheat 3,4 and 5 group chromosomes during wheat formation and the evolution of gene families in the different sub-genome is independent, which may relate to gene function.

Gene duplication is frequently revealed in plant genomes, resulting from polyploidization or through tandem and segmental duplication related to replication [30]. Here, we found 17 homologous gene groups with a copy on each of A, B and D homologous chromosome, and 7 gene pairs with a copy on only 2 of the 3 homologous chromosomes, while the other 13 genes were not found as homologs (Fig. 2, Additional file 3). Our results indicate

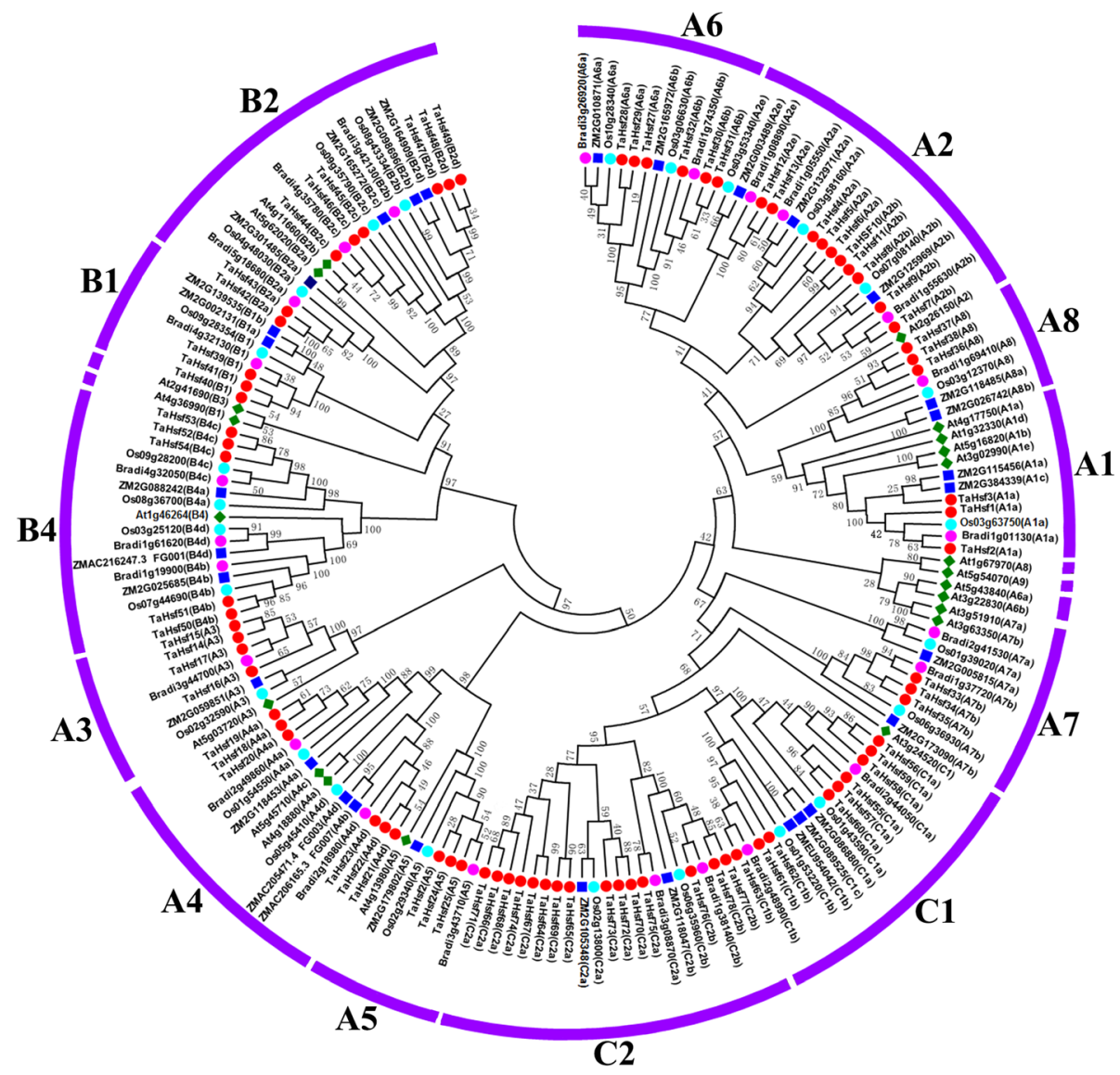

Fig. 1 Phylogenetic tree of Hsf proteins from wheat, Arabidopsis, rice, brachypodium and maize. The N-proximal regions (from the start of the DNA-binding domain to the end of the HR-A/B region) of Hsf proteins were used to construct an unrooted neighbor-joining tree with MEGA6.0 (with pairwise deletion and Poisson correct). For Hsf proteins of Arabidopsis (prefixed by AT), rice (prefixed by Os), Brachyposium (prefixed by Bradi) and maize (prefixed by ZM), both locus ID and subclass numbers are given. TaHsf proteins are marked in red 


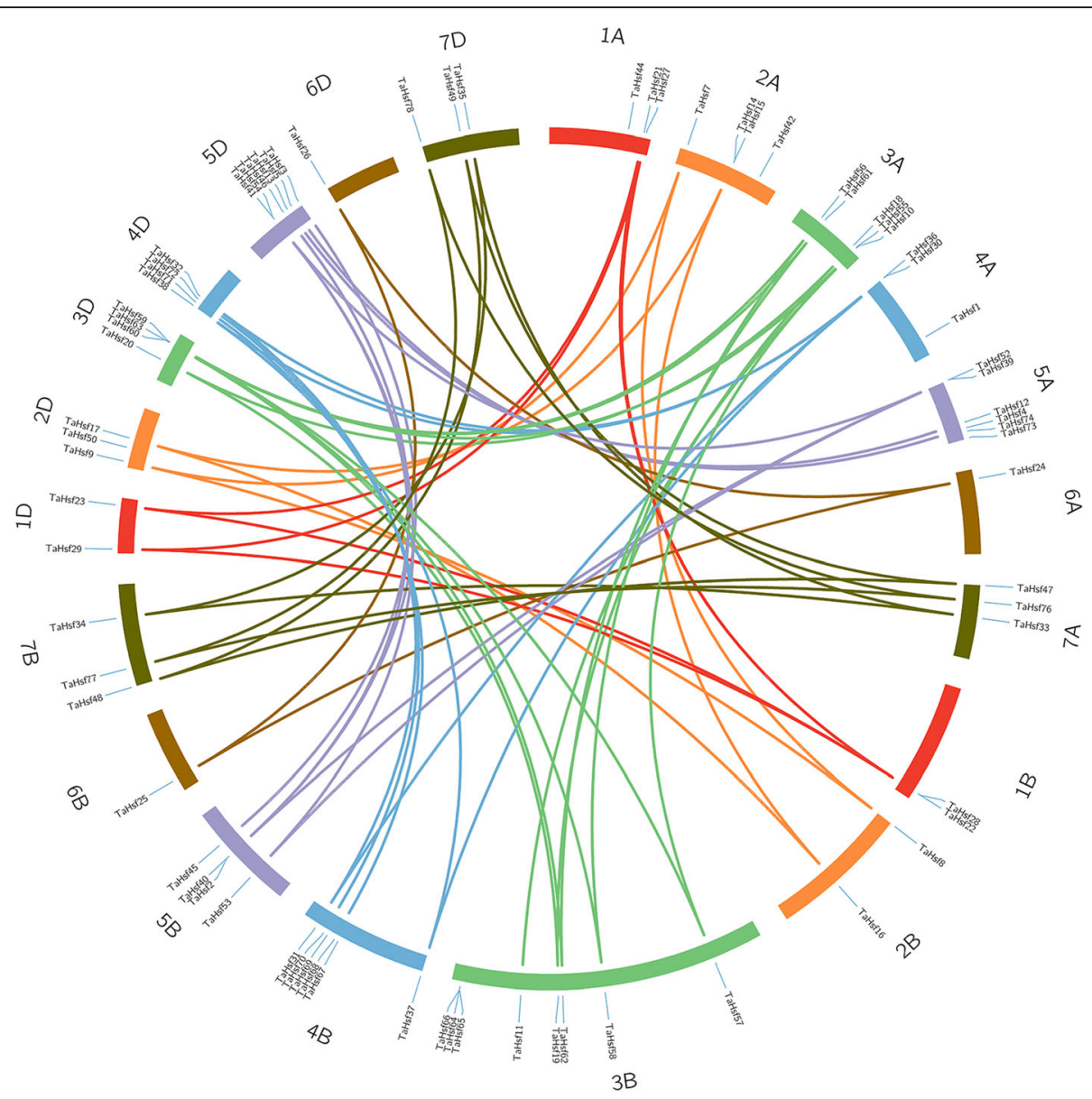

Fig. 2 Chromosomal localizations and the homologous TaHsf genes in wheat A, B and D sub-genomes

that gene loss may happen throughout the wheat $H s f$ gene family, leading to the loss of some homologous copies. Moreover, these homologous genes are clustered in group 3,4 and 5 chromosomes, which was in line with the above analysis of chromosome localization, suggesting that group 3, 4 and 5 chromosomes subjected less sequence loss and interaction impact compared to other homologous chromosome groups. In addition, 17 pairs of duplicated genes from different sub-genomes were also found, containing 3 duplication events in the same chromosome and 14 segmental duplication events between different chromosomes, indicating that the duplication events could play important roles in the extension of the Hsf genes in wheat genome (Fig. 3, Additional file 3).

\section{Phylogenetic analysis of Hsfs between the T. urartu, A. tauschii, and wheat orthologs}

We also identify the $H s f s$ gene in the diploid ancestors of wheat, $T$. urartu and $A$. tauschii, to investigate the change of $H s f$ number in transition from diploidy to hexaploidy within a given subgenome. Results showed that 16 and 15 putative $H s f s$ were identified in T. urartu and A. tauschii through our methods, respectively (Additional file 4). Total $16 \mathrm{~T}$. urartu-Hsfs, $25 \mathrm{~T}$. aestivum-A-Hsfs, $15 \mathrm{~A}$. tauschii-Hsfs, and $23 \mathrm{~T}$. aestivum-D-Hsfs gene sequences were applied to build gene trees. 16 pairs of T. urartuwheat A genome orthologs were mapped to T. urartu chromosomes with 2 on $1 \mathrm{~A}, 2$ on $2 \mathrm{~A}, 4$ on $3 \mathrm{~A}, 3$ on $4 \mathrm{~A}, 2$ on $5 \mathrm{~A}, 1$ on $6 \mathrm{~A}$ and 2 on 7A (Fig. 4). 15 pairs of $A$. tauschii-wheat $\mathrm{D}$ genome orthologs were mapped to A.tauschii chromosomes with 2 on 1D, 3 on 2D, 3 on 3D, 2 on 4D, 3 on 5D, 1 on 6D and 1 on 7D (Fig. 4). The majority of the orthologs (75 and $66.67 \%$ for $T$. urartu and A. tauschii, respectively) belonged to class $\mathrm{A}$, as expected due to the high proportional composition of this type (48.72\%) among the identified wheat $H s f$ genes. Moreover, the chromosome locations of the majority of wheat $H s f$ genes and their orthologs in T. urartu and A. tauschii corresponded to one another (Additional file 5).

\section{Modulatory network between TaHsf genes with other wheat genes}

In order to comprehend the interactions between TaHsfs and other wheat genes, the modulatory network of them 


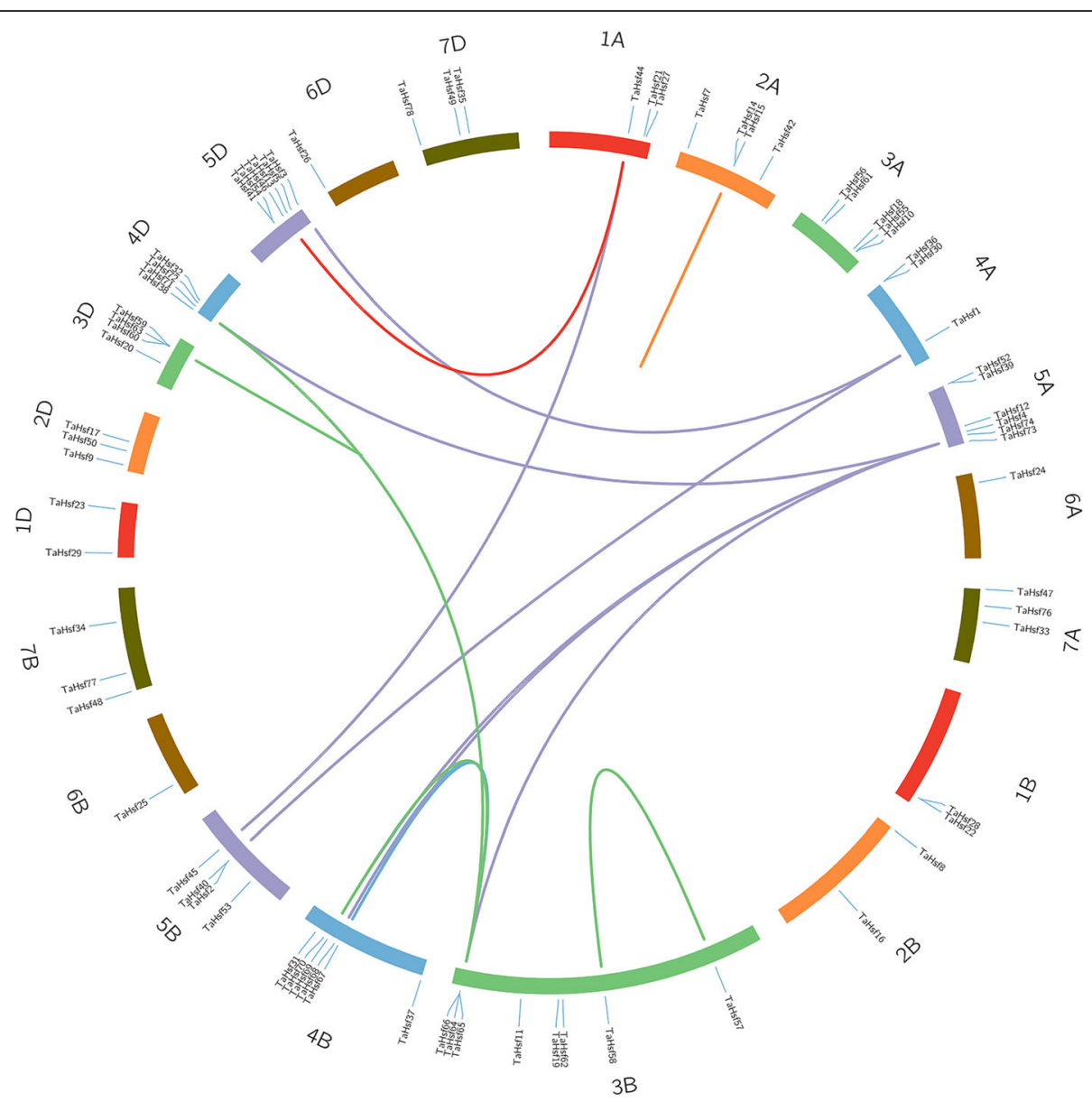

Fig. 3 Duplicated Hsf gene pairs identified in wheat. Seven homologous groups of wheat chromosomes are depicted in different colors. Duplicated gene pairs are depicted in corresponding colors and linked using lines with the corresponding color

(Fig. 5) was predicted via the orthology-based method [31]. Results showed that $15 \mathrm{TaHsfs}$ were shown to have homology with Arabidopsis genes and the 420 gene pairs of network interactions were found with the average of 28 gene per TaHsf, suggesting the TaHsfs were broadly engaged in the regulatory network and biological process in wheat. Among these, 292 genes interacted with TaHsfA and 128 genes interacted with TaHsfB. TaHsf16 (A3) was found to interact with 77 wheat genes, including Hsp81.4, ZF2, HBT and HSP90.1, suggesting it was mainly participated in response to stress, metal ion binding, cell differentiation and protein folding. TaHsf 18 $(A 4 a)$ was found to interact with 24 wheat genes, including ZAT6, STZ and S6K2, suggesting it was mainly engaged in metal ion binding, intracellular signal transduction and negative regulation of cell proliferation. TaHsf50 (B4b) was predicted to interact with 88 wheat genes, including $M Y B 15, M Y B 70, Z F P 2, F M A$, and $H B 31$, suggesting it is engaged primarily in the regulation of transcription, asmonic acid, metal ion binding and DNA binding. TaHsf44 (B2c) was found to interact with 30 wheat genes including AGC2-1, WRKY39, $B A G 6$ and $N F-Y C 2$, suggesting it is mainly engaged in defense response, calmodulin binding, response to heat and flower development (Additional files 6, 7). Moreover, GO and KEGG pathway descriptions of those interacting genes were analyzed to understand the potential function and pathway of the 15 TaHsfs (Fig. 6). The 15 TaHsf interacting genes were significantly enriched for transcription, DNA-templating, response to heat, transcription factor activity, sequence-specific DNA binding and calmodulin binding (Fig. 6a). Significantly enriched pathways included plant hormone signal transduction, $P I 3 K-A k t$ signaling pathway, and protein processing in endoplasmic reticulum (Fig. 6b).

\section{Tissue-specific expression patterns of TaHsf genes}

Using available RNA-seq data for five different tissues, the tissue specificity of the TaHsf genes was investigated to focus on the temporal and spatial expression patterns and putative functions of $H s f$ genes in wheat growth and development. According to FPKM values, we found that 


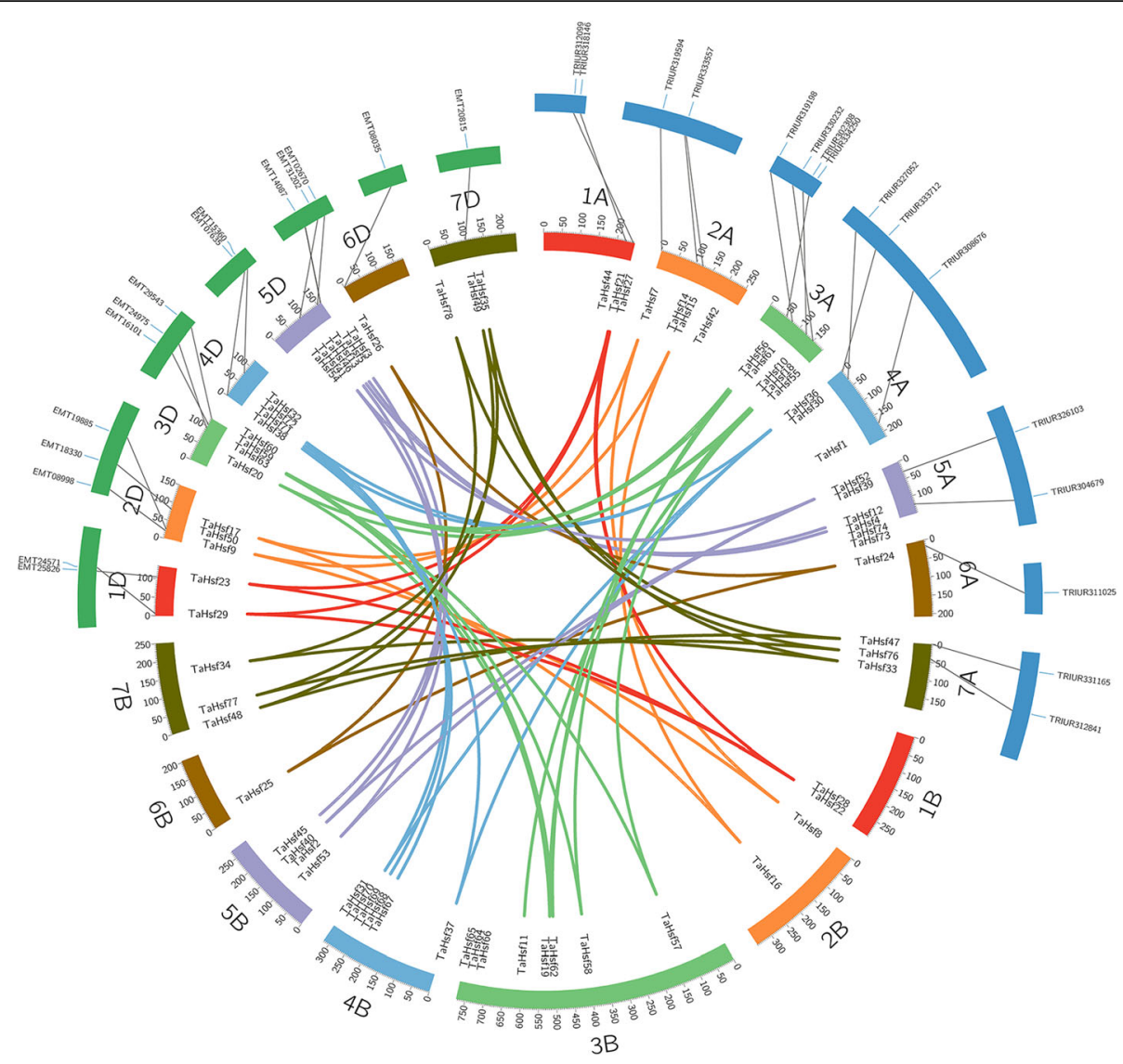

Fig. 4 Collinear analysis for the Hsf gene family among wheat, T.urartu and A.tauschii. The green annulus on the top left represent chromosomes of A. tauschii and the blue annulus on the top right represent chromosomes of T. urartu. Different colors represent seven homologous groups of wheat chromosomes. Homeologous genes of each group are linked by lines with corresponding color

the expression levels of the TaHsfs varied significantly in different tissues (Fig. 7). TaHsf10 (A2b), TaHsf15 (A3), TaHsf16 (A3), TaHsf17 (A3), TaHsf30 (A6b), TaHsf32 (A6b), TaHsf50 (B4b), TaHsf58 (C1a), TaHsf66 (C2a) and TaHsf72 (C2a) exhibit low expression abundance in endosperm, inner pericarp and outer pericarp, while TaHsf1 (A1a), TaHsf2 (A1a), TaHsf3 (A1a), TaHsf4 (A2a), TaHsf8 (A2b), TaHsf9 (A2b), TaHsf2O (A4a), TaHsf21 (A4d), TaHsf36 (A8) and TaHsf41 (B1) had high expression abundances. Furthermore, the expression levels of the TaHsfs varied significantly in different grain layers over development (Additional file 1: Figure S2).

Expression patterns of TaHsf genes under abiotic stresses To study the roles of TaHsf genes in response to abiotic stresses, expression of all TaHsf genes in response to drought, heat, and Cd stress was investigated using RNA sequencing data. All 46 wheat $H s f$ genes revealed different expression patterns under these dynamic conditions. Among them, the expression levels of TaHsf2 (A1a) and TaHsf21 (A4d) were both down-regulated under drought, heat, drought and heat stresses, while the expression of TaHsf4 (A2a), TaHsf15 (A3), TaHsf16 (A3), TaHsf17 (A3), TaHsf28 (A6a) and TaHsf41 (B1) was upregulated (Additional file 1: Figure $S 3$ ). According to our RNA sequencing data (Additional file 8) [31], expression levels of TaHsf3 (A1a), TaHsf4 (A2a), TaHsf5 (A2a), TaHsf16 (A3), TaHsf18 (A4a), TaHsf2O (A4a), TaHsf31 $(A 6 b)$ and TaHsf32 (A6b) were up-regulated under $\mathrm{Cd}$ stress, while the expression of TaHsf7 (A2b), TaHsf8 (A2b), TaHsf9 (A2b), TaHsf26 (A5) and TaHsf50 (B4b) was down-regulated (Fig. 8).

\section{Verification of the expression of TaHsf in wheat under cd stress by qRT-PCR}

According to the expression analysis based on diverse RNA sequencing data above, we obtained an overview of expressed TaHsfs under various agriculturally-relevant stressors. To further verify these results we selected a subset of these TaHsfs to detect their expression levels in root under Cd stress through qRT-PCR. Results showed that compared with H17CK group, levels of TaHsf3 (A1a), TaHsf4 (A2a), TaHsf5 (A2a), TaHsf16 (A3), TaHsf18 (A4a), TaHsf2O (A4a), TaHsf31 (A6b) and 


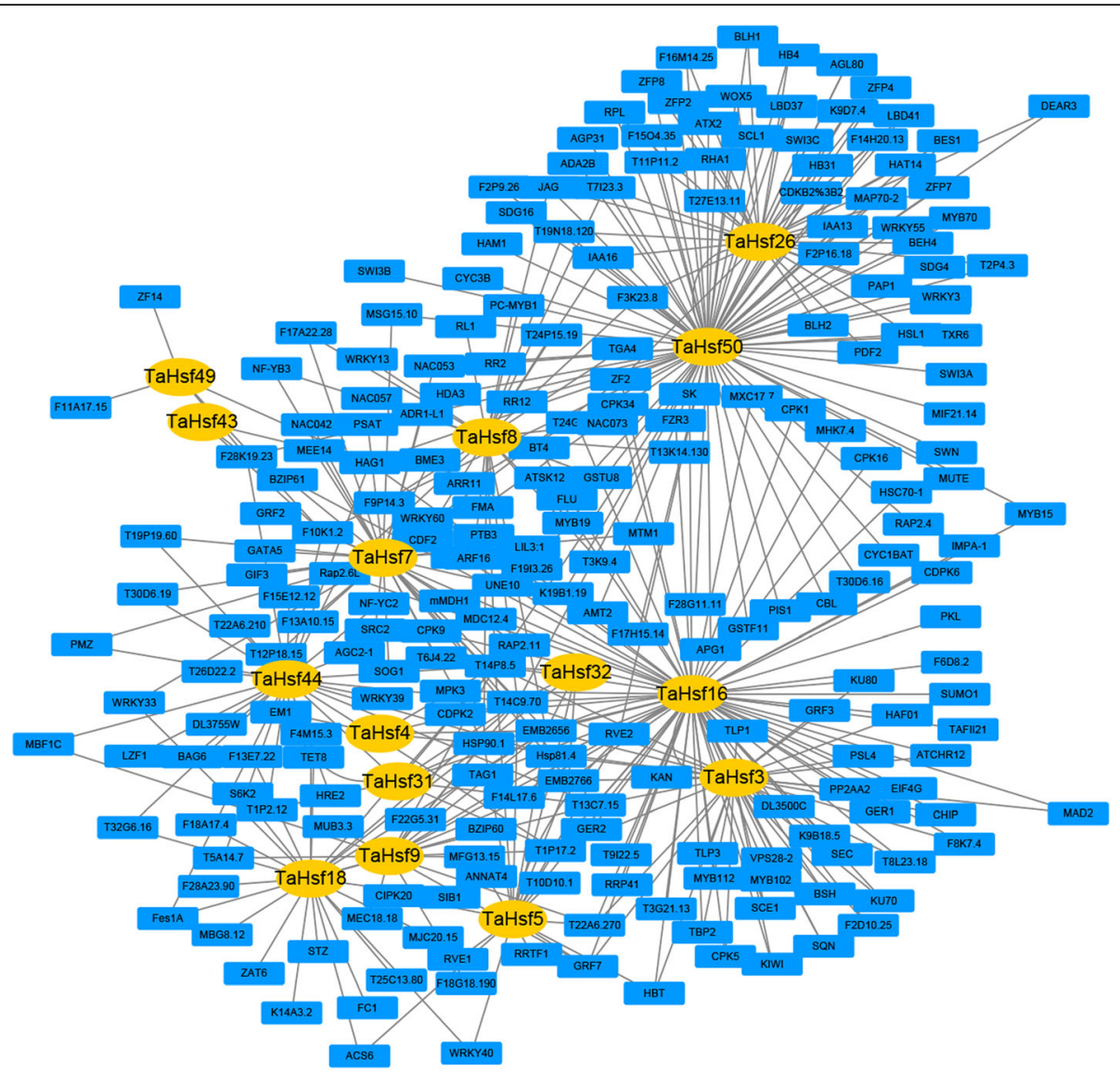

Fig. 5 An interaction network of TaHsf genes in wheat based on the orthologs in Arabidopsis. Fifteen TaHsfs were found to have homology with Arabidopsis genes and the 420 gene pairs of network interactions

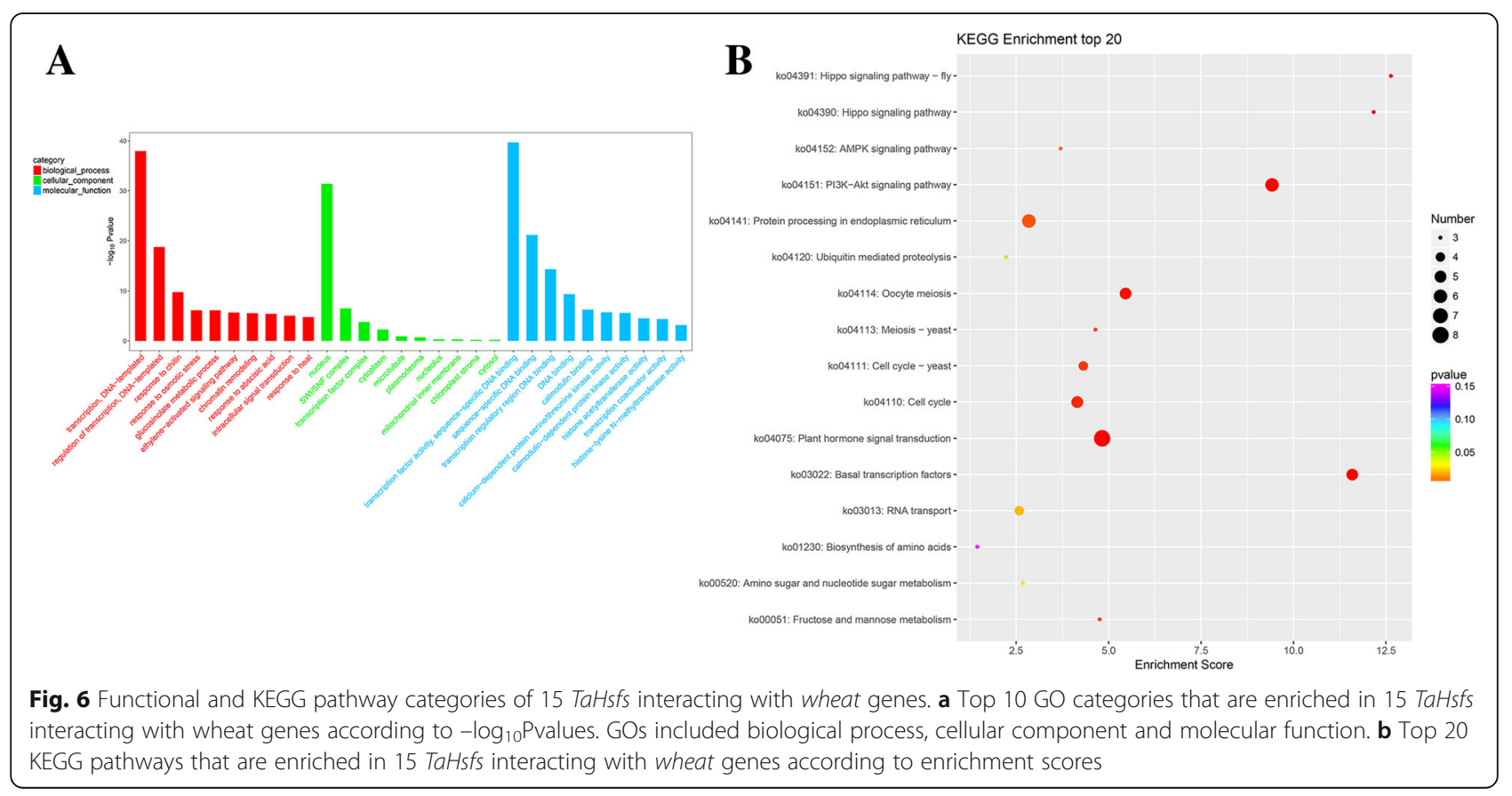




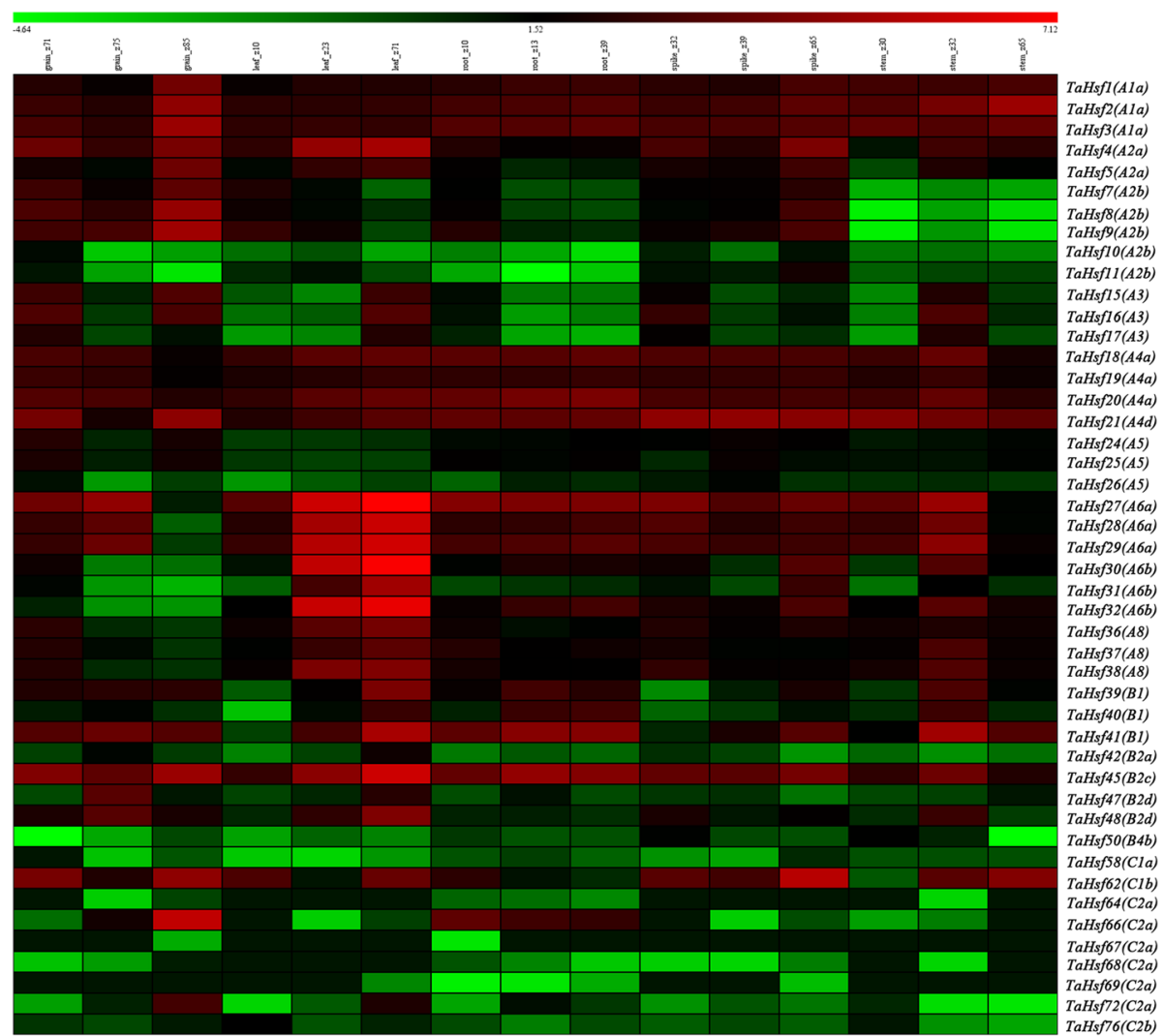

Fig. 7 Heat map of the expression profiles of 46 TaHsf genes in five different tissues (grain, leaf, root, spike and stem). Log2 transformed FPKM values were used to create the heat map. The red or green colors stand for the higher or lower relative abundance of each transcript in each sample. $Z$ represent Zadoks scale, a decimal code for the growth stages of cereals. $P$-value $<0.05$ were regarded as statistically significant

TaHsf32 (A6b) were significantly increased, while levels of TaHsf7 (A2b), TaHsf8 (A2b), TaHsf9 (A2b), TaHsf26 $(A 5)$ and TaHsf50 (B4b) were significantly decreased $(P<0.05$, Fig. 9$)$. The qRT-PCR results were highly consistent with that of RNA sequencing data, confirming that it is reasonable to use RNA sequencing data to evaluate the expression level of transcripts in wheat $\mathrm{Cd}$-response.

\section{Discussion}

A growing body of evidence shows that Hsfs play essential roles in plant developmental and defense processes $[16,32-35]$. Due to growing numbers of quality genomes available, putative functions of $H s f$ family genes have been predicted in many species, from the model plants Arabidopsis [13], rice [5] and maize [36], now to other crops, such as apple [21], Chinese cabbage [37], Chinese white pear [38] and pepper [39]. However, despite the global impact of wheat, as well as the importance of environmental $\mathrm{Cd}$ contamination, there has been limited investigation into the molecular basis of $\mathrm{Cd}$ accumulation, and the $H s f$ family in wheat.

Here we took advantage of the high quality wheat reference genome, to first identify $78 \mathrm{Hsf}$ wheat genes and to characterize these bioinformatically (Table 1). A first contrast lies on the sheer quantity of these genes in wheat: while we identify 78 in wheat, there are only 21 $H s f s$ in Arabidopsis, 25 in rice, 30 in maize, 29 in Chinese white pear and 25 in apple [5, 13, 36, 38]. The vast majority of $H s f s$ can be categorized into three classes: A, $\mathrm{B}$ and $\mathrm{C}$. The quantity of class $\mathrm{A}$ in Arabidopsis, rice, maize, Chinese white pear and apple are 15, 13, 16, 19 and 16, respectively. Class B Hsfs amount to 5, 8, 9, 8 and 7 , in the five plants respectively. Finally, class $C$ is represented by 1, 9, 4, 2 and 2, respectively. In contrast, of 78 putative wheat $H s f$ genes, 38 belonged to class A, 16 to class $B$ and 24 to class $C$. Thus class $C$ is relatively expanded in wheat in contrast to these other genomes.

We next investigated occurrences of possible gene duplication, which contributes differentially to the extension of specific gene families in plant genomes, and results from polyploidization or tandem and segmental duplication related [30, 40, 41]. In wheat, we found that homologous genes are gathered in group 3, 4 and 5 chromosomes, which was in line with the above analysis of chromosome localization. These results indicated that compared to other homologous chromosome groups, 


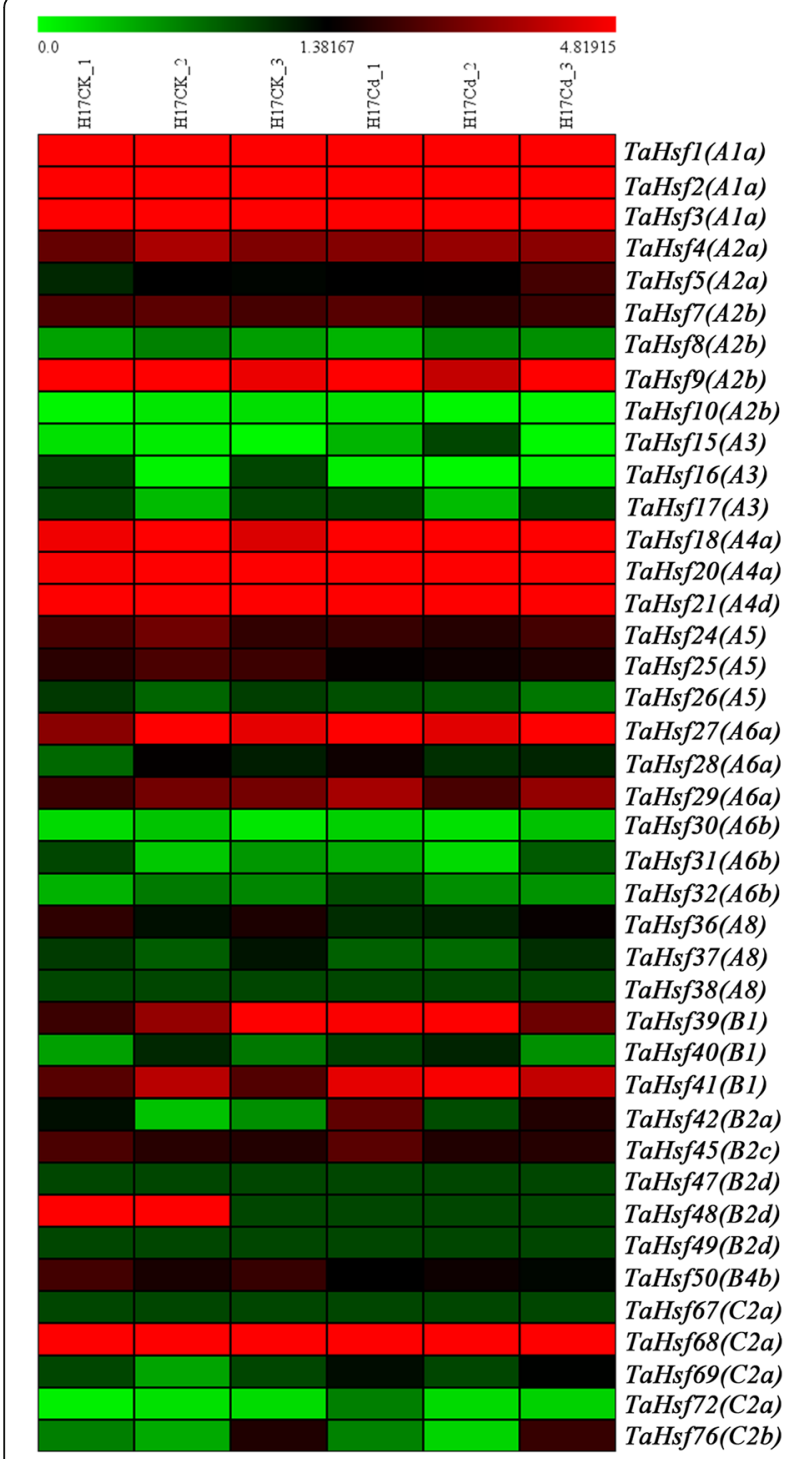

Fig. 8 Heat map of the expression profiles of TaHsf genes under $\mathrm{Cd}$ treatment. FPKM values were used to create the heat map. The red or green colors indicate the higher or lower relative abundance or each transcript in each sample

group 3, 4 and 5 chromosomes suffered less sequence loss and interaction impact. Three duplication events with the same chromosome and 14 segmental duplication events between various chromosomes were identified, suggesting that in wheat genome, the duplication events could play important roles in the extension of the $H s f$ cascade genes. A previous study indicated that more than $90 \%$ of the enhancement in regulatory genes in the Arabidopsis lineage were facilitated via genome duplications [42]. Compared with tandem duplications, segmental $H s f$ gene duplications were more often. This situation appeared in Arabidopsis, maize, poplar [21, 22, 36], and also in wheat.
Our phylogenetic analysis indicated that compared with Arabidopsis, maize and rice, brachypodium Hsfs were nearer to wheat Hsf proteins, which was in line with broader classifications. Identification of $H s f$ genes in wheat and its diploid ancestors, T. urartu and $A$. tauschii, which suggesting that the number of Hsf in a known subgenome was increased in transition from diploidy to hexaploidy (for A subgenome, 16 to 25 genes, and for D subgenome, 15 to 23 genes). These results further indicate that gene gain happened broadly during the formation of hexaploid [27].

Moreover, protein-protein regulatory interactions were constructed to provide inference of mechanisms of life activities and to explore potential biological functions for unknown proteins. Results showed that TaHsf18 (A4a) interacts with 24 wheat genes, including ZAT6, $S T Z$ and $S 6 K 2$, suggesting it was mainly engaged in metal ion binding, intracellular signal transduction, and the negative regulation of cell proliferation. A previous study indicated that ZAT6 coordinately activates the expression of phytochelatin synthesis-related gene and positively modulate $\mathrm{Cd}$ accumulation and tolerance by directly targeting GSH1 in Arabidopsis [43]. HsfA4a was also engaged in cadmium tolerance in wheat [19], suggesting it might be involved in metal ion binding via interacting with ZAT6 to further play a role in cadmium tolerance in wheat. TaHsf50 (B4b) interacts with 88 wheat genes, including MYB15, MYB70, ZFP2, FMA, and $H B 31$, suggesting it is involved in regulation of transcription, regulation of jasmonic acid, metal ion binding and DNA binding. It has been reported that MYB15 is required for the defense-induced synthesis of G-rich lignin and the constitutive synthesis of the coumarin metabolite scopoletin, both of which contribute to disease resistance against a hemibiotrophic bacterial pathogen [44]. TaHsf44 (B2c) was found to interact with 30 wheat genes including $A G C 2-1$, WRKY39, BAG6 and $N F-Y C 2$, suggesting it is engaged in defense response, calmodulin binding, response to heat and flower development. AtBAG6 can induce programmed cell death in yeast and plants [45]. Aspartyl protease-mediated cleavage of BAG6 plays an important role in autophagy and fungal resistance in plants [46]. GO analysis showed that 15 TaHsfs interacted genes were significantly enriched for transcription, DNA-templating, response to heat, transcription factor activity, sequence-specific DNA binding and calmodulin binding. It has been reported that $H s f$ family has a unique role as master modulators of thermotolerance, and were essential for plants survival under serious heat stress $[9,47]$.

Furthermore, we characterize wheat Hsf genes that expression throughout tissues and development stages. Many of these genes were highly expressed across development. For example, TaHsf2, 3, 20, 17 and 45 were high 


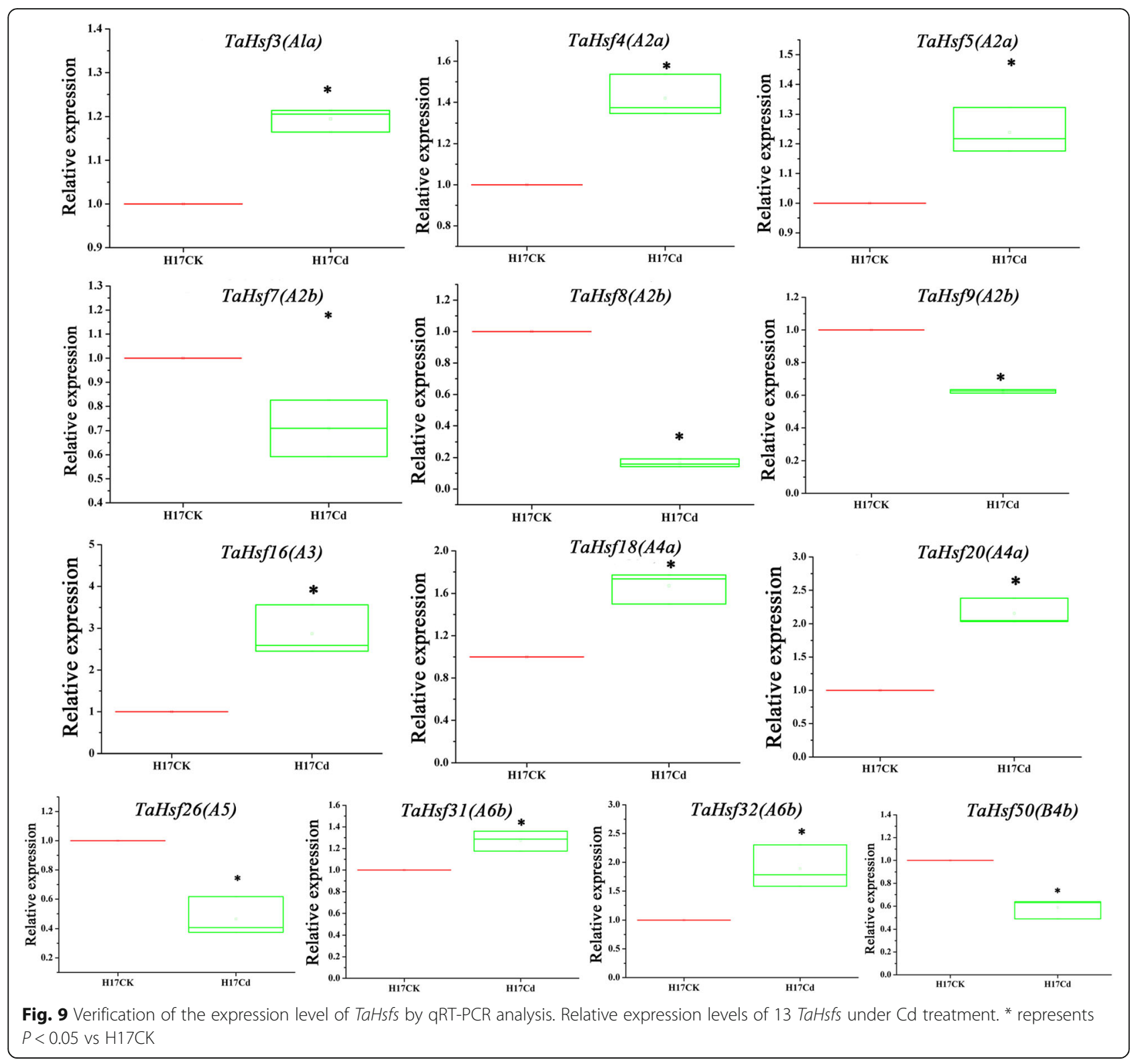

expressed in roots, stems, leaves, spikes and grains including whole endosperm, starchy endosperm, transfer cells and aleurone layer, as well as seed coats during different developmental stages. It has been reported that Hsfs were involved in plant growth and development $[9,16]$. Our results further indicated that $H s f$ genes play important regulatory roles in wheat growth, development and reproductive processes.

In addition, we comprehensively analyzed the expression levels of $H s f$ genes in response to drought, heat and $\mathrm{Cd}$ stresses to predict potential roles. The expression of most $H s f$ genes were differentially regulated in response to a given stress, which strongly suggests that they may be vital stress response genes. A previous study indicated that $H s f s$ are involved in responses to the abiotic stress as heat, cold, salt, drought and cadmium [13, 17, 19]. Our results first comprehensively illustrate that $H s f$ genes likely play important regulatory roles in wheat $\mathrm{Cd}$ stress response. Therefore, these genes stand as strong functional candidates for followup research into $\mathrm{Cd}$ stress in wheat.

\section{Conclusion}

We present the first comprehensive identification and characterization of the wheat $H s f$ gene family. Through the latest available wheat genome information, total 78 putative wheat $H s f$ gens were identified through a genome-wide search, and categorized into class A, B and $\mathrm{C}$ subfamilies based on conserved motifs. Chromosome localizations, gene structures, conserved protein motifs, 
and phylogenetic relationship of these TaHsfs were comprehensively analyzed and strongly supported these classifications. Moreover, the gene duplication and homologous genes between wheat $\mathrm{A}, \mathrm{B}$ and $\mathrm{D}$ sub-genome were also surveyed. Expression profiles of these TaHsfs through development and under various abiotic stresses were surveyed and provide strong functional candidates for followup work. Finally, through qRT-PCR analysis, 13 TaHsf genes were selected to verify their expression level in wheat under $\mathrm{Cd}$ stress, which provide top candidates for further functional analysis of $H s f$ genes in response to wheat $\mathrm{Cd}$ stress.

\section{Methods}

Identification and classification of $H s f$ gene family in wheat

The $H s f$ gene family was identified following the method as described by Wang et al. with some modifications [48]. First, to construct a local protein database, all the wheat ( $T$. aestivum $L$.) protein sequences available were downloaded from the Ensemble database (http://plants. ensembl.org/index.html). Then, the database were searched with 100 known $H s f$ gene sequences collected from A. thaliana (21), O. sativa (25), B. distachyon (24) and $Z$. mays (30) using the local BLASTP program with an e-value of le- 5 and identity of $50 \%$ as the threshold. Moreover, a self-blast of these sequences was performed to remove redundancy, the physical localizations of all candidate $H s f$ genes were checked and redundant sequences with the same chromosome location were rejected. Furthermore, all obtained $H s f$ protein sequences were analyzed to detect DBD domains and coiled-coil structures by the SMART and MARCOIL programs (SMART: http://smart.embl-heidelberg.de/, MARCOIL: http://toolkit.tuebingen.mpg.de/marcoil). Those protein sequences lacking the DBD domain or a coiled-coil structure were removed. Finally, to verify the existence of all the obtained sequences, BLASTN similarity searches against the wheat ESTs deposited in the NCBI database were performed. The theorectical pI (isoelectric point) and $\mathrm{Mw}$ (molecular weight) of the putative Hsf from $T$. aestivum $L$ were calculated using compute $\mathrm{pI} / \mathrm{Mw}$ tool online (http://web.expasy.org/compute_pi/), respectively. Classification of the three different groups A, B and $\mathrm{C}$ was based on structural characteristics and phylogenetic comparisons $[49,50]$.

\section{Gene structure construction, protein domain and motif analysis}

Gene structure information were obtained from the Ensemble plants database (http://plants.ensembl.org/index.html). Conserved domains annotation was performed using Pfam (http://pfam.xfam.org/search), SMART (http://smart.emblheidelberg.de/) and Heatster online tools [39]. All full- length amino acid sequences of the TaHsfs were used to identify conserved domain motifs by the Multiple Em for Motif Elicitation (MEME) tool [51]. The parameters were set as follows: maximum numbers of different motifs, 30; minimum motif width, 4; maximum motif width, 50.

\section{Chromosomal locations and gene duplication}

Genes were mapped onto chromosomes by identifying their chromosomal position provided in the wheat genome database. Gene duplication events of Hsf genes in wheat were investigated based on the following three criteria: (a) the alignment covered $>80 \%$ of the longer gene; (b) the aligned region had an identity $>80 \%$ [52]. In order to visualize the duplicated regions in the $T$. aestivum genome, lines were drawn between matching genes using Circos-0.67 program (http://circos.ca/).

\section{Phylogenetic analysis}

The N-terminal Hsf protein sequences containing the DBD and HR-A/B regions and parts of the linker between these two regions from A. thaliana, O. sativa, $B$. distachyon, $Z$. mays and $T$. aestivum $L$. were performed for multiple alignments by CLUSTALW and the results of alignment were used to construct phylogenetic tree using the NJ method in MEGA (version 6.0) [53]. Bootstrap test method was adopted and the replicate was set to 1000 .

\section{Analysis of the TaHsf family orthologs in $T$. urartu and $A$. tauschii}

The wheat- $T$. aestivum, wheat-T. urartu and wheat- $A$ .tauschii Hsf genes were used to construct phylogenetic trees using neighbor-joining method with 1000 bootstrap replicates. According to these orthologous $H s f$ genes, a collinear map of the T. urartu-wheat A genome and $A$. tauschii-wheat $\mathrm{B}$ genome was created using genome visualization tool CIRCOS according to these orthologous Hsf genes. The locations of $H s f$ orthologous genes on the chromosomes of T. urartu and A. tauschii were obtained from the database published by Ling et al. [23] and Jia et al. [54], respectively.

\section{Network interaction analysis}

The interaction network involving the TaHsf genes was based on the orthologous genes between Wheat and Arabidopsis using the AraNet V2 tool (http://www.inetbio.org/aranet/) [48]. Enrichment analysis was implemented by BiNGO, a cytoscape plugin, for gene ontology analysis and identifying processes and pathways of specific gene sets. Over-represented GO full categories were identified with a significance threshold of 0.01 . 
The TaHsf gene expression analysis by RNA-seq data To study the expression of TaHsf genes in different organs and response to stress, the wheat expression database (http://wheat.pw.usda.gov/WheatExp/) was used The FPKM (fragments per kilobase of transcript per million fragments mapped) value was calculated for each $H s f$ gene, the $\log 2$ transformed values of the TaHsf genes were used for heat map generation. $P$-values $<0.05$ were taken as statistically significant thresholds [55].

\section{Plant materials, growth conditions, and treatments}

The plant of wheat cultivar Chuanyu17, a high-Cdaccumulating cultivar, was planted in growth chambers at $23 \pm 1{ }^{\circ} \mathrm{C}$ with a photoperiod of $16 \mathrm{~h}$ light $/ 8 \mathrm{~h}$ dark. One-week-old seedlings were treated with 0 (H17CK) and $100 \mu \mathrm{M} \mathrm{CdCl} 2$ for $24 \mathrm{~h}(\mathrm{H} 17 \mathrm{Cd})$. Roots from the plants with similar size were harvested separately and washed three times with deionized water. All the plant samples from three biological replicates were frozen in liquid nitrogen immediately and stored at $-80{ }^{\circ} \mathrm{C}$ for RNA extraction.

\section{RNA extraction and real-time quantitative RT-PCR (qRT- PCR) analysis}

Total RNA was extracted from roots of Chuanyu17 in H17CK and H17Cd groups using TRIzol Reagent (Invitrogen, USA) according to the manufacturer's instructions. RNA was quantified by using NanoDrop1000 and RNA integrity was checked by electrophoresis. First strand cDNA was synthesized using HiScript IIQ RT SuperMix (Vazyme, R223-1). The primers used in the qRT-PCR analyses are listed in Additional file 9. $\beta$ actin was used as an internal control. The qRT-PCR was carried out using QuantiFast ${ }^{\circ} \mathrm{SYBR}^{\circ}$ Green PCR kit (Qiagen, 204,054) according to the manufacturer's instructions. Each treatment was repeated three times. The expression levels were calculated from the $2^{-\Delta \Delta \mathrm{Ct}}$ value $[\Delta \Delta \mathrm{Ct}=(\mathrm{CT}$ target $/ \mathrm{Cd}-\mathrm{CT}$ actin/Cd $-(\mathrm{CT}$ target/control - $\left.\mathrm{CT}_{\text {actin/control }}\right]$ [45].

\section{Additional files}

Additional file 1: Figure S1. Motifs identified by MEME tools in Wheat Hsfs. Thirty motifs (1-30) were identified and indicated by different color. Motif location and combined $p$-value were represented. Motif 9 was found in TaHsf5, 6, 9, 10, 11, 13, 17, 18, 20, 23, 27, 28, 30, 31, 32, 45, 46, $52,56,59,60,64,65,66,68,73$ and 75 which was covered by other motifs. Figure S2. Heat map of the expression profiles of TaHsf genes in different grain layers and a developmental timecourse. Log2 transformed FPKM values were used to establish the heat map. The red or green colors stand for the higher or lower relative abundance of each transcript in each sample. $P$-value $<0.05$ were regarded as statistically significant. DPA means days post-anthesis. Figure $\mathbf{S 3}$. Heat map of the expression profiles of TaHsf genes under drought and heat stress treatments. Log2 transformed FPKM values were used to create the heat map. The red or green colors indicate the higher or lower relative abundance or each transcript in each sample. $P$-value $<0.05$ were regarded as statistically significant. (PDF $580 \mathrm{~kb}$ )

Additional file 2: Motif sequences identified by MEME tools. Motif numbers corresponded to the motifs in Additional file 1: Figure S1. (XLSX $10 \mathrm{~kb})$

Additional file 3: The homologous TaHsf genes in wheat A, B and D sub-genomes and the Duplicated genes pairs identified in wheat (XLSX $11 \mathrm{~kb})$

Additional file 4: The list of the putative Hsf genes for A.tauschii and T.urartu (XLSX $11 \mathrm{~kb}$ )

Additional file 5: Details of TaHsfs and corresponding orthologs Hsfs in T.urartu and A.tauschii (XLSX $11 \mathrm{~kb}$ )

Additional file 6: The detail of 15 TaHsf orthologous genes in Arabidopis thaliana (XLSX $10 \mathrm{~kb}$ )

Additional file 7: Detail information of Network of TaHsf with other genes (XLSX $40 \mathrm{~kb}$ )

Additional file 8: Expression profiles of TaHsf in wheat under $\mathrm{Cd}$ stress (XLSX $13 \mathrm{~kb}$ )

Additional file 9: The Primers for TaHsfs. (XLSX $10 \mathrm{~kb}$ )

\section{Abbreviations}

AHA: Activator motifs; Cd: Cadmium; DBD: DNA binding domain; FPKM: Fragments per kilobase of transcript per million fragments mapped; GO: Gene ontology; HR-A/B: Oligomerizationn domain; Hsf: Heat shock transcription factor; HSPs: Heat shock proteins; MEME: Multiple Em for Motif Elicitation; MT: Mutant; NES: Nuclear export signal; NLS: Nuclear localization signal; qRT-PCR: real-time quantitative RT-PCR

Acknowledgements

We thank Xian Fu, Pengfei Xiang, Liangliang Ju and Xiaoyun Huang for technical support.

Authors' contributions

$M Z$ and $Y W$ contributed to the experimental design and manuscript drafting $M Z, S G Z, L Z, R L$ and JL performed the research. MZ, CHZ and ZHL performed bioinformatics analysis. MZ, LL and CPL contributed reagents/materials/ analysis tools. LY supported the revision of the manuscript and drafted the manuscript revision with $\mathrm{MZ}$ and YW. All authors read and approved the final manuscript.

\section{Funding}

This work was supported by the "13th Five-year Plan" for National Key Research and Development (Grant No. 2016YFD0102000). LY acknowledges support from the European Research Council (ERC) under the European Union's Horizon 2020 research and innovation programme [grant number ERC-StG 679056 HOTSPOT]. The funding bodies had no role in the design of the study, collection, analysis, or interpretation of data, or in writing the manuscript.

\section{Availability of data and materials}

The dataset and materials presented in the investigation is available by request from the corresponding author.

Ethics approval and consent to participate

Not applicable.

\section{Consent for publication}

Not applicable.

\section{Competing interests}

The authors declare that they have no competing interests.

\section{Author details}

${ }^{1}$ Chengdu Institute of Biology, Chinese Academy of Sciences, No.9, section 4 of South RenMin Road, Wuhou District, Chengdu 610041, Sichuan, China. ${ }^{2}$ University of Chinese Academy of Sciences, Beijing 100049, China. ${ }^{3}$ School of Life Sciences, University of Nottingham, Nottingham NG7 2RD, UK. 
Received: 3 October 2018 Accepted: 31 May 2019

Published online: 18 June 2019

\section{References}

1. Hartl FU, Hayer-Hartl M. Molecular chaperones in the cytosol: from nascent chain to folded protein. Science. 2002;295:1852-8.

2. Young JC, Barral JM, Ulrich Hartl F. More than folding: localized functions of cytosolic chaperones. Trends Biochem Sci. 2003;28:541-7.

3. Wu C. Heat shock transcription factors: structure and regulation. Annu Rev Cell Dev Biol. 1995;11:441-69.

4. Sarkar A. Heat shock factor gene family in rice: genomic organization and transcript expression profiling in response to high temperature, low temperature and oxidative stresses. Plant Physiol Biochem. 2009:47:785.

5. Guo J, Wu J, Ji Q, Wang C, Luo L, Yuan Y, Wang Y, Wang J. Genome-wide analysis of heat shock transcription factor families in rice and Arabidopsis. J Genet Genomics. 2008:35:105-18.

6. Nover L, Bharti K, Doring P, Mishra SK, Ganguli A, Scharf KD. Arabidopsis and the heat stress transcription factor world: how many heat stress transcription factors do we need? Cell Stress Chaperones. 2001;6:177-89.

7. Kotak S, Larkindale J, Lee U, Von KP, Vierling E, Scharf KD. Complexity of the heat stress response in plants. Curr Opin Plant Biol. 2007;10:310-6.

8. Baniwal SK, Bharti K, Chan KY, Fauth M, Ganguli A, Kotak S, et al. Heat stress response in plants: a complex game with chaperones and more than twenty heat stress transcription factors. J Biosci. 2004;29:471-87.

9. Scharf KD, Berberich T, Ebersberger I, Nover L. The plant heat stress transcription factor ( $\mathrm{Hsf}$ ) family: structure, function and evolution. Biochim Biophys Acta. 2012;1819:104-19.

10. Lyck R, Harmening U, Hohfeld I, Treuter E, Scharf KD, Nover L. Intracellular distribution and identification of the nuclear localization signals of two plant heat-stress transcription factors. Planta. 1997;202:117-25.

11. Heerklotz D, Doring P, Bonzelius F, Winkelhaus S, Nover L. The balance of nuclear import and export determines the intracellular distribution and function of tomato heat stress transcription factor HsfA2. Mol Cell Biol. 2001; 21:1759-68.

12. Kotak S, Port M, Ganguli A, Bicker F, von Koskull-Doring P. Characterization of C-terminal domains of Arabidopsis heat stress transcription factors (Hsfs) and identification of a new signature combination of plant class a Hsfs with AHA and NES motifs essential for activator function and intracellular localization. Plant J. 2004:39:98-112.

13. Swindell WR, Huebner M, Weber AP. Transcriptional profiling of Arabidopsis heat shock proteins and transcription factors reveals extensive overlap between heat and non-heat stress response pathways. BMC Genomics. 2007:8:125.

14. Almoguera C, Rojas A, Díazmartín J, Prietodapena P, Carranco R, Jordano J. A seed-specific heat-shock transcription factor involved in developmental regulation during embryogenesis in sunflower. J Biol Chem. 2002;277:43866.

15. Díaz-Martín J, Almoguera C, Prieto-Dapena P, Espinosa JM, Jordano J. Functional interaction between two transcription factors involved in the developmental regulation of a small heat stress protein gene promoter. J Am Chem Soc. 2005:139:1483.

16. Kotak S, Vierling E, Baumlein H, von Koskull-Doring P. A novel transcriptional cascade regulating expression of heat stress proteins during seed development of Arabidopsis. Plant Cell. 2007;19:182-95.

17. Nishizawa A, Yabuta Y, Yoshida E, Maruta T, Yoshimura K, Shigeoka S. Arabidopsis heat shock transcription factor A2 as a key regulator in response to several types of environmental stress. Plant J. 2006:48:535-47.

18. Miller G, Mittler R. Could heat shock transcription factors function as hydrogen peroxide sensors in plants? Ann Bot. 2006;98:279-88.

19. Shim D, Hwang JU, Lee J, Lee S, Choi Y, An G, Martinoia E, Lee Y. Orthologs of the class A4 heat shock transcription factor HsfA4a confer cadmium tolerance in wheat and rice. Plant Cell. 2009:21:4031-43.

20. Chauhan $H$, Khurana N, Agarwal P, Khurana P. Heat shock factors in rice (Oryza sativa L.): genome-wide expression analysis during reproductive development and abiotic stress. Mol Genet Genomics. 2011;286:171-87.

21. Filomena G, Gea G, Sanja B, Celestina M. Heat shock transcriptional factors in Malus domestica: identification, classification and expression analysis. BMC Genomics. 2012;13:639.

22. Wang F, Dong Q, Jiang H, Zhu S, Chen B, Xiang Y. Genome-wide analysis of the heat shock transcription factors in Populus trichocarpa and Medicago truncatula. Mol Biol Rep. 2012;39:1877-86.
23. Ling HQ, Zhao S, Liu D, Wang J, Sun H, Zhang C, et al. Draft genome of the wheat A-genome progenitor Triticum urartu. Nature. 2013:496:87-90.

24. Dubcovsky J, Dvorak J. Genome plasticity a key factor in the success of polyploid wheat under domestication. Science. 2007;316:1862-6.

25. Matsuoka Y. Evolution of polyploid triticum wheats under cultivation: the role of domestication, natural hybridization and allopolyploid speciation in their diversification. Plant Cell Physiol. 2011;52:750-64.

26. Wang M, Wang S, Xia G. From genome to gene: a new epoch for wheat research? Trends Plant Sci. 2015;20:380-7.

27. Mayer KFX, Rogers J, Doležel J, Pozniak C, Eversole K, Feuillet C, et al. A chromsome-based draft sequence of the hexaploid bread wheat (Triticum aestivum) genome. Science. 2014;345:1251788.

28. Wicker T, Mayer KF, Gundlach H, Martis M, Steuernagel B, Scholz U, et al. Frequent gene movement and pseudogene evolution is common to the large and complex genomes of wheat, barley, and their relatives. Plant Cell. 2011;23:1706-18.

29. Brenchley R, Spannagl M, Pfeifer M, Barker GL, D'Amore R, Allen AM, et al. Analysis of the bread wheat genome using whole-genome shotgun sequencing. Nature. 2012;491:705-10.

30. Zhang J. Evolution by gene duplication: an update. Trends Ecol Evol. 2003; 18:292-8.

31. Zhou M, Zheng S, Liu R, Lu J, Lu L, Zhang C, et al. Comparative analysis of root transcriptome profiles between low- and high-cadmium-accumulating genotypes of wheat in response to cadmium stress. Funct Integr Genomics. 2019;19:281-94

32. Kumar M, Busch W, Birke H, Kemmerling B, Nurnberger T, Schoffl F. Heat shock factors $\mathrm{HsfB} 1$ and $\mathrm{HsfB} 2 \mathrm{~b}$ are involved in the regulation of Pdf1.2 expression and pathogen resistance in Arabidopsis. Mol Plant. 2009;2:152-65.

33. Pérez-Salamó I, Papdi C, Rigó G, Zsigmond L, Vilela B, Lumbreras V, et al. The heat shock factor A4A confers salt tolerance and is regulated by oxidative stress and the mitogen-activated protein kinases MPK3 and MPK6. Plant Physiol. 2014;165:319.

34. Giorno F, Woltersarts M, Grillo S, Scharf KD, Vriezen WH, Mariani C. Developmental and heat stress-regulated expression of HsfA2 and small heat shock proteins in tomato anthers. J Exp Bot. 2010;61:453-62.

35. Ulrike B, Albihlal WS, Tracy L, Fryer MJ, Sparrow PAC, François R, et al. Arabidopsis HEAT SHOCK TRANSCRIPTION FACTORA1 boverexpression enhances water productivity, resistance to drought, and infection. J Exp Bot. 2013;64:3467.

36. Lin YX, Jiang HY, Chu ZX, Tang XL, Zhu SW, Cheng BJ. Genome-wide identification, classification and analysis of heat shock transcription factor family in maize. BMC Genomics. 2011;12:76.

37. Song XM, Huang ZN, Duan WK, Ren J, Liu TK, Li Y, Hou XL. Genome-wide analysis of the bHLH transcription factor family in Chinese cabbage (Brassica rapa ssp. pekinensis). Mol Genet Genomics. 2014;289:77-91.

38. Qiao X, Li M, Li L, Yin H, Wu J, Zhang S. Genome-wide identification and comparative analysis of the heat shock transcription factor family in Chinese white pear (Pyrus bretschneideri) and five other Rosaceae species. BMC Plant Biol. 2015;15:12

39. Guo M, Lu JP, Zhai YF, Chai WG, Gong ZH, Lu MH. Genome-wide analysis, expression profile of heat shock factor gene family (CaHsfs) and characterisation of CaHsfA2 in pepper (Capsicum annuum L.). BMC Plant Biol. 2015;15:151.

40. Freeling M. Bias in plant gene content following different sorts of duplication: tandem, whole-genome, segmental, or by transposition. Annu Rev Plant Biol. 2009;60:433-53.

41. Wang $Y$, Wang $X$, Tang $H$, Tan $X$, Ficklin SP, Feltus FA, Paterson AH. Modes of gene duplication contribute differently to genetic novelty and redundancy, but show parallels across divergent angiosperms. PLoS One. 2011;6:e28150.

42. Maere S, De Bodt S, Raes J, Casneuf T, Van Montagu M, Kuiper M, Van de Peer Y. Modeling gene and genome duplications in eukaryotes. Proc Natl Acad Sci U S A. 2005;102:5454-9.

43. Chen J, Yang L. Zinc-Finger Transcription Factor ZAT6 Positively Regulates Cadmium Tolerance through the Glutathione-Dependent Pathway in Arabidopsis. Plant Physiol. 2016;171:707-19.

44. Chezem WR, Memon A, Li F-S, Weng J-K, Clay NK. SG2-type R2R3-MYB transcription factor MYB15 controls defense-induced lignification and basal immunity in Arabidopsis. Plant Cell. 2017;29:1907-26.

45. Kang CH, Jung WY, Kang YH, Kim JY, Kim DG, Jeong JC, et al. AtBAG6, a novel calmodulin-binding protein, induces programmed cell death in yeast and plants. Cell Death Differ. 2006;13:84-95. 
46. Li Y, Kabbage M, Liu W, Dickman MB. Aspartyl protease-mediated ceavage of BAG6 is necessary for autophagy and fungal resistance in plants. Plant Cell. 2016;28:233-47.

47. Mishra SK, Tripp J, Winkelhaus S, Tschiersch B, Theres K, Nover L, Scharf KD. In the complex family of heat stress transcription factors, HsfA1 has a unique role as master regulator of thermotolerance in tomato. Genes Dev. 2002;16:1555-67.

48. Wang M, Yue H, Feng K, Deng P, Song W, Nie X. Genome-wide identification, phylogeny and expressional profiles of mitogen activated protein kinase kinase kinase (MAPKKK) gene family in bread wheat (Triticum aestivum L.). BMC Genomics. 2016;17:668.

49. von Koskull-Doring P, Scharf KD, Nover L. The diversity of plant heat stress transcription factors. Trends Plant Sci. 2007;12:452-7.

50. Harrison CJ, Bohm AA, Nelson HC. Crystal structure of the DNA binding domain of the heat shock transcription factor. Science. 1994;263:224-7.

51. Bailey TL, Williams N, Misleh C, Li WW. MEME: discovering and analyzing DNA and protein sequence motifs. Nucleic Acids Res. 2006;34:W369.

52. Wang J, Sun N, Deng T, Zhang L, Zuo K. Genome-wide cloning, identification, classification and functional analysis of cotton heat shock transcription factors in cotton (Gossypium hirsutum). BMC Genomics. 2014 15:961.

53. Tamura K, Stecher G, Peterson D, Filipski A, Kumar S. MEGA6: molecular evolutionary genetics analysis version 6.0. Mol Biol Evol. 2013;30:2725-9.

54. Jia J, Zhao S, Kong X, Li Y, Zhao G, He W, et al. Aegilops tauschii draft genome sequence reveals a gene repertoire for wheat adaptation. Nature. 2013;496:91-5.

55. Pearce S, Vazquez-Gross H, Herin SY, Hane D, Wang Y, Gu YQ, Dubcovsky J. WheatExp: an RNA-seq expression database for polyploid wheat. BMC Plant Biol. 2015;15:299.

\section{Publisher's Note}

Springer Nature remains neutral with regard to jurisdictional claims in published maps and institutional affiliations.

Ready to submit your research? Choose BMC and benefit from:

- fast, convenient online submission

- thorough peer review by experienced researchers in your field

- rapid publication on acceptance

- support for research data, including large and complex data types

- gold Open Access which fosters wider collaboration and increased citations

- maximum visibility for your research: over 100M website views per year

At $\mathrm{BMC}$, research is always in progress.

Learn more biomedcentral.com/submissions 